\title{
Effects of Internal Circulation and Particle Mobility during Nanofluid Droplet Evaporation
}

\author{
Y. Wei ${ }^{1}, \mathrm{~W}$. Deng ${ }^{2}$, and Ruey-Hung Chen ${ }^{3, \$}$ \\ ${ }^{1}$ Department of Mechanical and Aerospace Engineering \\ University of Central Florida, Orlando, FL 32816-2450 \\ ${ }^{2}$ Department of Mechanical Engineering \\ Virginia Polytechnic Institute \& State University, Blacksburg, VA 24016 \\ ${ }^{3}$ Department of Mechanical and Aerospace Engineering \\ New Mexico State University, Las Cruces, NM
}

\begin{abstract}
The effect of internal circulation on evaporation of fluid droplets containing nano-sized particles is analytically investigated, where internal circulation is caused by viscous effects at the liquid-gas interface in the convective environment. The competing time scales of liquid diffusion, convection, and particle diffusion are first analyzed as influenced by gas phase velocity, relative viscosities of gas and liquid phases, and the droplet size. The results reveal the importance of internal recirculation for droplets of practical sizes. To demonstrate the role of internal circulation plays on particle distribution and shell formation during the evaporation process, a symmetric Hill vortex and strong circulation are assumed for solving a one-dimensional governing equation, which to yield (i) the particle redistribution during the evaporation process, (ii) the time and the size upon shell formation (i.e., the end of the first-stage evaporation/drying) due to inclusion of particles at the droplet surface, and (iii) internal particle distribution that forecast possible morphologies of particle aggregates once the drying process is complete. These results are found to be dependent upon (a) the relative time scales of liquid diffusion and particle mobility (the effect of Lewis number, $L e$ ), and (b) the relative importance or evaporation rate $(K)$ and particle mobility (the effect of Peclet number, $\mathrm{Pe}$ ). Comparisons with previous results without internal circulation are made, as related to the time for shell formation and possible morphologies. Such comparisons reveal some distinctly different, and surprising, phenomena during and at the end of the first-stage evaporation.
\end{abstract}

Key words - evaporation, nanofluid, nano-particles, Peclet number, Lewis number, particle mobility

${ }^{\$}$ Corresponding author; chenrh@nmsu.edu 


\section{Introduction}

In a previous article by these authors [1], evaporation behavior of droplets containing nano-sized particles was studied using a model that is constructed based on, and supported by, the existing experimental result. Of particular interest are the phenomena, evolution of droplet size, and evaporation rate constant prior to the shell formation, when the particles forms a compact shell at the droplet surface. Both the droplet and the particle are assumed to be spherical, without particle collisions and breakup. As the droplet evaporation proceeds, increasingly more particles become "included" on its surface [2], reducing the effective liquid surface area for evaporation. Effects of Peclet number $(\mathrm{Pe})$, a non-dimensional ratio of particle diffusion time to the droplet lifetime (or the inverse of the evaporation rate), is shown to play an important role. A large/small Pe suggests that the particles has a relatively short/long time to redistribute within the regressing droplet and the inclusion rate is large/small. Other parameters, such as initial particle concentration, also play roles. The model predictions reveal a few important aspect of evaporation od droplets containing nano-sized particles: (1) there exists deviation from the classical $d^{2}$-law for pure fluid droplet evaporation and the deviation is increasingly significant with increased $P e$ and initial particle concentration. Such deviations arise from the fact that particles included one the droplet surface reduce liquid surface area from which evaporation takes place; therefore the $d^{2}$-law based on pure liquid surface properties ceases to hold. (2) The time to shell formation (also the end of the firststage evaporation [1]) decreases, and shell diameter increases, with increased $P e$ and initial particle concentration; the reverse occurs with decreased $P e$ and initial particle concentration. (3) As a consequence, there exist a limiting combination of $P e$ and initial concentration to where there is instant shell formation and the entire evaporation takes place only after the liquid diffuses to the surface of the shell. The effect of contact angle is also discussed, which plays a role determining the effective liquid surface area for evaporation.

Consequently, the nanofluid properties, namely $P e$ and initial particle concentration together plays an important role in affecting droplet evaporation rate even prior to shell formation, and thus

affecting the deviation from the $d^{2}$-law [3-5], mainly arising from the inclusion process. (As a comparison, a multi-component droplets with soluble solids, the deviation from the $d^{2}$-law is attributed to variable liquid properties [6,7].) Similarly, it also affects the size of the shell 
formation and the possible morphology of the final dried aggregate; this is because the short/long time for shell formation (and thus larger shell diameter) implies hollow/compact aggregates. On the other hand, two competing rate processes - particle diffusion rate and the droplet evaporation rate - play dynamic roles during evaporation. However, such a Peclet number neglects the effect of liquid circulation within the droplet, which is commonly caused by convective current around the droplet in applications such as spray drying and pharmaceutical manufacturing. In [1], the effect of internal circulation within the droplet was mentioned and was modeled phenomenologically by reducing effective values of $P e$ but not explicitly modeled. The convective current generates shear stresses on the droplet surface that, in turns, causes flow within the droplet in such a way as to satisfy the shear stress continuity. The liquid circulation enhances both heat transfer coefficient and modify the temperature distribution within the droplet and further causes deviation from the $d^{2}$-law [7-9]. It is expected that internal circulation will modify the particle distribution with the droplet and how the particles might be reaching the droplet surface and be "included" [2].

The internal circulation is expected to increase the effective particle diffusion coefficient, similar to the eddy viscosity concept adopted in some turbulent flow studies. This leads to the concept of effective Peclet number (denoted by $P e_{\text {eff }}$ for this study), as adopted in [9-11] for multicomponent droplet evaporation. On the other hand, with the circulatory flow, gradients of particle concentration would be set up differently from those found in droplet without internal circulation. As a consequence, particle diffusion might not occurs in a single direction, that is, radially inward as found in [1] and the particle inclusion rate would have to be determined by the combined convection and diffusion transport processes.

This paper aims to develop a model to predict the effect of internal circulation on how particles redistribute within the nanofluid droplet during the evaporation process and, as a consequence, to predict the characteristic scales of time and size, particularly those at the end of the first stage drying (i.e., when the particle shell forms). Special attention is paid to the effects of (1) relative diffusivities of the liquid and the particle, the latter being Einstein-Stokes diffusivity (a form of Lewis number), (2) relative effects of evaporation and particle diffusion rate (a form of a Peclet number), and (3) the effect of the initial particle concentration. 


\section{Model Development}

\subsection{Characteristic times of particle diffusion, internal circulation, and droplet evaporation}

Internal circulation within a liquid droplet in a convective environment results from the viscous interaction at the liquid-gas interface. The internal circulation within a droplet was theoretically studied by Hill [12] more than a century ago. In that study, an axisymmetric Hill's vortex, was used to describe the internal flow resulting from a laminar external flow. For the external flow, Hadamard [13] solved the basic hydrodynamics of the liquid droplet with creeping viscous flow passing by. Hill's vortices were later visualized by Garner et al. [14, 15]. More recently, Wong et al. [16] provided evidence of internal recirculation inside large hydrocarbon droplets in high temperature gas streams. These investigators captured the transient internal temperature distribution that is caused by internal droplet dynamics. Internal circulation was also visualized in acoustically levitated droplets [17], which are often used for investigating heat and mass transfer of stationary droplets. For particles contained in a droplet, their mobility (in addition to diffusion described by Einstein-Stokes equation) and redistribution during the droplet regression are affected by such internal circulation.

For vaporizing droplets with internal circulation, the redistribution of particles within them are affected by both the Einstein-Stokes diffusion and convection. The mechanism and models of internal circulation of liquid droplets has been described in some studies [18,19]. Perhaps because of the small Reynolds number associate with typically small diameters of droplets, creeping flow analysis has been frequently adopted in order to theoretically predict the strength of the internal circulation and the flow patterns inside the liquid droplet [13]. Consider a uniform, axi-symmetric, slow viscous gas flow passing around a droplet. The stream function inside the liquid droplet, $\psi_{i}$, is written as

$$
\psi_{i}=\frac{1}{4} U_{\infty} \sin ^{2} \theta\left(\frac{1}{1+\sigma}\right) r^{2}\left({\frac{r}{r_{s}^{2}}}^{2}-1\right)
$$

where 


$$
\sigma=\mu_{\text {liquid }} / \mu_{\text {gas }}
$$

and $r$ is the radial distance from the droplet center, $r_{s}$ the droplet radius, $U_{\infty}$ is the velocity of the gas flow far away from the droplet, $\mu_{o}$ and $\mu_{i}$ are, respectively, the dynamic viscosities of fluids outside and inside the droplet, and $\theta$ is measured from the incoming flow, as shown in Fig. 1. The velocity $v_{i}(r, \theta)$ inside the droplet is found with Eqn. (1) to be

$$
v_{i}(r, \theta)=\frac{1}{r \sin \theta}\left(\frac{\partial \psi_{i}}{\partial r}\right)_{\theta}=\frac{\sin \theta}{2}\left(\frac{\sigma}{1+\sigma}\right) \frac{U_{\infty}}{r_{s}^{2}}\left(2 r^{2}-r_{s}^{2}\right)
$$

where the derivative is evaluated at an arbitrary angle $\theta$.

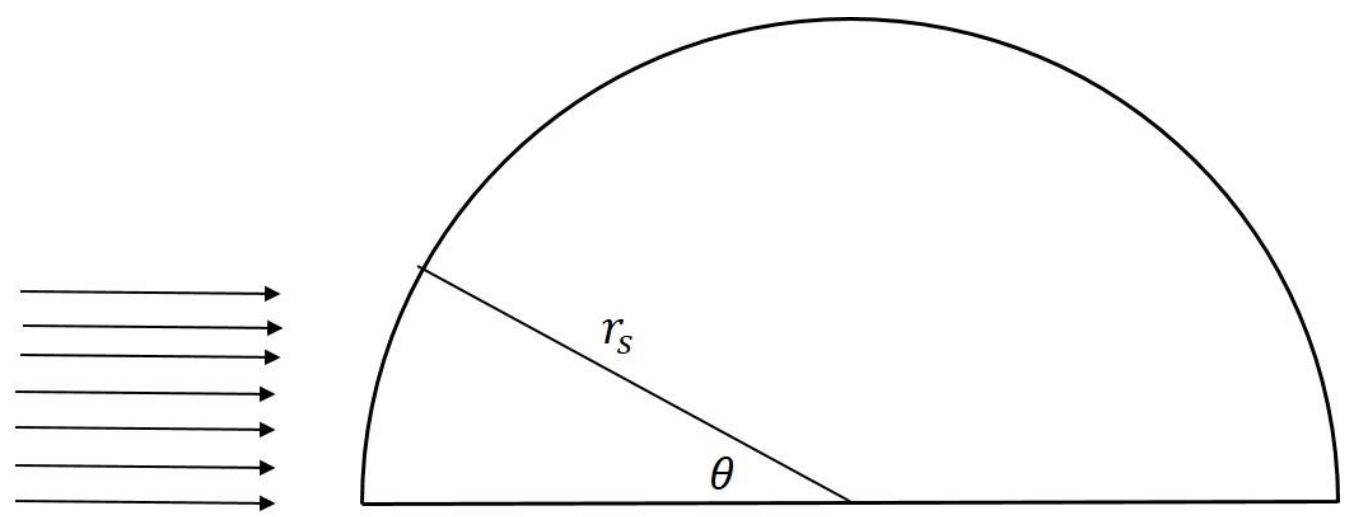

Figure 1 - Schematic of the upper hemisphere of the droplet and incoming flow, also showing the droplet radius $r_{s}$ and angle $\theta$.

The evaluation of $\psi_{i}$ shows that the flow field inside the liquid droplet is symmetric to the axis of the external flow, with several streamlines as shown in Fig. 2, which is adopted from [20]. Equation (3) suggests that there exists a stagnation ring inside the droplet, described by $r=r_{s} / \sqrt{2}$. Due to the viscous effect, this stagnation point lies along $\pi / 2<\theta<\pi$ The velocity of the liquid is also zero at the front and rear stagnation points, $\theta=0$ and $\theta=\pi$ at $r=r_{s}$. 


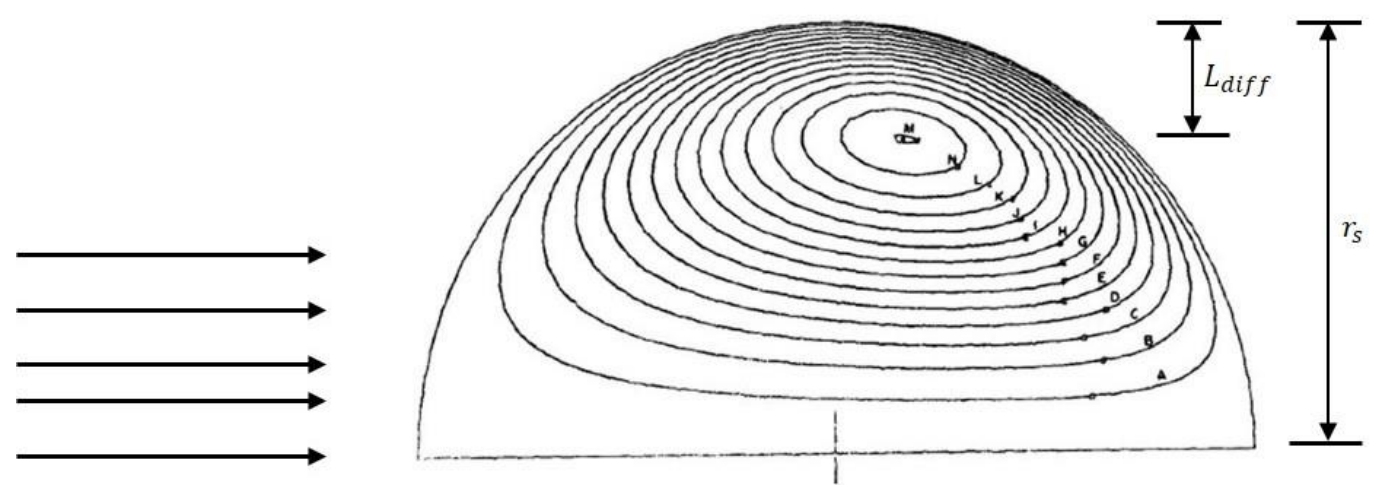

Figure 2 - Streamline pattern inside a water droplet for free-stream $\operatorname{Re}=30$ (Leclair et al. 1972); $r_{s}=d_{\mathrm{o}} / 2$.

The particles transport process is driven by the Einstein-Stokes diffusion, with the diffusivity given as

$$
D_{\mathrm{pl}}=k_{\mathrm{B}} T / 6 \pi \eta r_{\mathrm{p}}
$$

where $k_{\mathrm{B}}, T, \eta$, and $r_{\mathrm{p}}$ are Boltzmann constant, temperature, liquid viscosity, and the particle radius, respectively. Without internal circulation, the characteristic length for particle diffusion is $r_{s}$. With internal circulation, the characteristic particle diffusion length is reduced to be $L_{\text {diff }}(=$ $\left.[1-1 / \sqrt{2}] r_{s}\right)$, as described in Fig. 2. The characteristic time of particle diffusion from within the droplet to its surface is

$$
\tau_{\text {diff }}=\frac{L_{\text {diff }}^{2}}{D_{\mathrm{pl}}}=\frac{\left(1-\frac{\sqrt{2}}{2}\right)^{2} r_{\mathrm{s}}^{2}}{D_{\mathrm{pl}}}=\frac{0.086 r_{\mathrm{s}}^{2}}{D_{\mathrm{pl}}} \sim \frac{0.02 d_{\mathrm{o}}^{2}}{D_{\mathrm{pl}}}
$$

where $d_{\mathrm{o}}$ is the droplet diameter.

On the other hand, the characteristic time of evaporation is calculated using the $d^{2}$-law for droplet evaporation, which is 


$$
d^{2}=d_{\mathrm{o}}^{2}-K t
$$

where $d$ and $d_{\mathrm{o}}$ are the instantaneous and initial droplet diameters, respectively, and $K$ is the evaporation coefficient having the dimension of diffusivity and determined by the properties of the liquid and the surroundings. The characteristic time for evaporation is therefore

$$
\tau_{\text {evap }}=\frac{d_{\mathrm{o}}^{2}}{K}=\frac{4 r_{\mathrm{s}}^{2}}{K}
$$

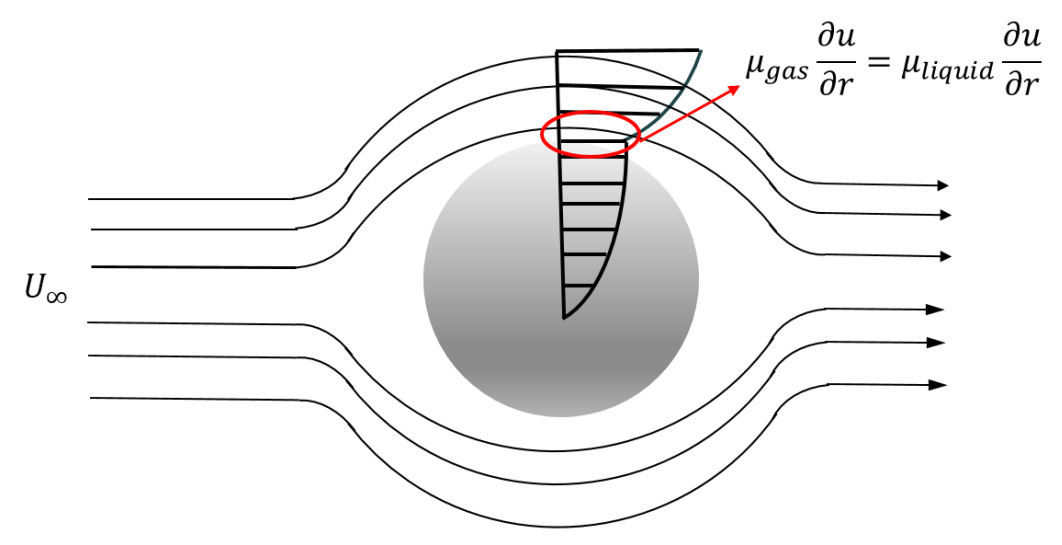

Figure 3 - Schematic of a droplet in a flow with relevant parameters.

The characteristic time of internal circulation is

$$
\tau_{\text {conv }}=\frac{d_{\mathrm{o}}}{V_{\mathrm{i}}}
$$

where $V_{\mathrm{i}}$ is the characteristic velocity of the internal flow. To obtain the value of $V_{\mathrm{i}}$, consider a droplet in a free stream with velocity $U_{\infty}$, with the shear boundary condition, $\mu_{\text {gas }}(\partial u / \partial r)_{\text {gas }}=$ 
$\mu_{\text {liquid }}(\partial u / \partial r)_{\text {liquid, }}$, as shown in Fig. 3, with $\mu_{\text {gas }}$ and $\mu_{\text {liquid }}$ being the dynamic viscosity of the surrounding gas and liquid For the droplet shown in Fig. 3 the drag force is

$$
F=3 \pi \mu_{\mathrm{gas}} U_{\infty} d_{\mathrm{o}}
$$

The shear force at the gas liquid interface is

$$
F=\mu_{\text {liquid }} A \frac{\partial u}{\partial r}=\mu_{\text {liquid }}\left(\pi d_{\mathrm{s}}^{2}\right) \frac{V_{\mathrm{i}}}{d_{\mathrm{s}} / 2}
$$

where $A$ is the droplet surface area. Combining equations (9) and (10) yields

$$
V_{\mathrm{i}}=\frac{3}{2} \frac{\mu_{\text {gas }}}{\mu_{\text {liquid }}} U_{\infty}
$$

For water droplet in air flow

$$
V_{\mathrm{i}} \sim \frac{\mu_{\text {gas }}}{\mu_{\text {liquid }}} U_{\infty} \sim \frac{1}{1000} U_{\infty}
$$

\subsection{A case study of various time scales}

To illustrate the effect of effective Peclet number $\left(P e_{\text {eff }} \equiv \tau_{\text {conv }} / \tau_{\text {evap }}\right)$, consider a special case where a single water droplet containing nano-particle suspensions in air flow at weak forced convection under standard atmospheric conditions, with particle diameter $d_{\mathrm{p}}=100 \mathrm{~nm}$ (resulting in $\left.D_{\mathrm{pl}}=10^{-11} \mathrm{~m}^{2} / \mathrm{s}\right), U_{\infty}=5.5 \mathrm{~cm} / \mathrm{s}$, and $K=10^{-9} \mathrm{~m}^{2} / \mathrm{s}$. These conditions leads to an external flow Reynolds number $R e=5$. The values of $\tau_{\text {diff }}, \tau_{\text {conv }}$ as functions of $d_{\mathrm{s}}$ under these given conditions are plotted in Fig. 4. There are two intersections between the various transport time scales. The time scales of $\tau_{\text {evap }}$ and $\tau_{\text {conv }}$ are the same at approximately $d_{\mathrm{s}}=18 \mu \mathrm{m}$, while the time scales of $\tau_{\text {diff }}$ and $\tau_{\text {conv }}$ coincides at approximately $d_{\mathrm{o}}=9 \mu \mathrm{m}$. For droplet size larger than $18 \mu \mathrm{m}$, 


$$
\tau_{\text {diff }}>\tau_{\text {evap }}>\tau_{\text {conv }}
$$

As can be seen in Fig. 4 , both $\tau_{\text {evap }}$ and $\tau_{\text {conv }}$ are about twice smaller than $\tau_{\text {diff }}$ for $d_{\mathrm{o}}>18 \mu \mathrm{m}$, where internal circulation dominates over Einstein-Stokes diffusion for particle redistribution within the evaporating droplet. On the other hand, $P e_{\text {eff }}=\tau_{\text {conv }} / \tau_{\text {evap }}<1$ and the particles have time for redistribution and avoiding being captured (or "included") at the droplet surface, resulting in a relatively solid particle aggregate at the time of shell formation. As the result in Fig. 4 shows, for $d_{\mathrm{o}} \gg 18 \mu \mathrm{m} P e_{\mathrm{eff}} \ll 1$ one can expect a very solid particle aggregate. For $18 \mu \mathrm{m}>d_{\mathrm{o}}>$ $8.9 \mu \mathrm{m}$,

$$
\tau_{\text {diff }}>\tau_{\text {conv }}>\tau_{\text {evap }}
$$

where internal circulation dominates over Einstein-Stokes diffusion for particle redistribution. In this droplet size range because $\tau_{\text {conv }}>\tau_{\text {evap }}$ the particle might be transported in such a way they cannot escape inclusion in the early stage of evaporation. Furthermore, $P e_{\text {eff }}=\tau_{\text {conv }} / \tau_{\text {evap }} \approx$ $O(1)$ suggests that inclusion will take place even in the earlier stage of evaporation. For $d_{\mathrm{o}}<$ $9 \mu \mathrm{m}$,

$$
\tau_{\text {conv }}>\tau_{\text {diff }}>\tau_{\text {evap }}
$$

In this case the particle redistribution is mainly achieved by Einstein-Stokes diffusion that dominates over internal circulation. However, because $P e_{\text {eff }}=\tau_{\text {diff }} / \tau_{\text {evap }}>1$ particles are captured at the droplet surface before significant redistribution takes place by diffusion. The final particle aggregate will likely be hollow in the center. 


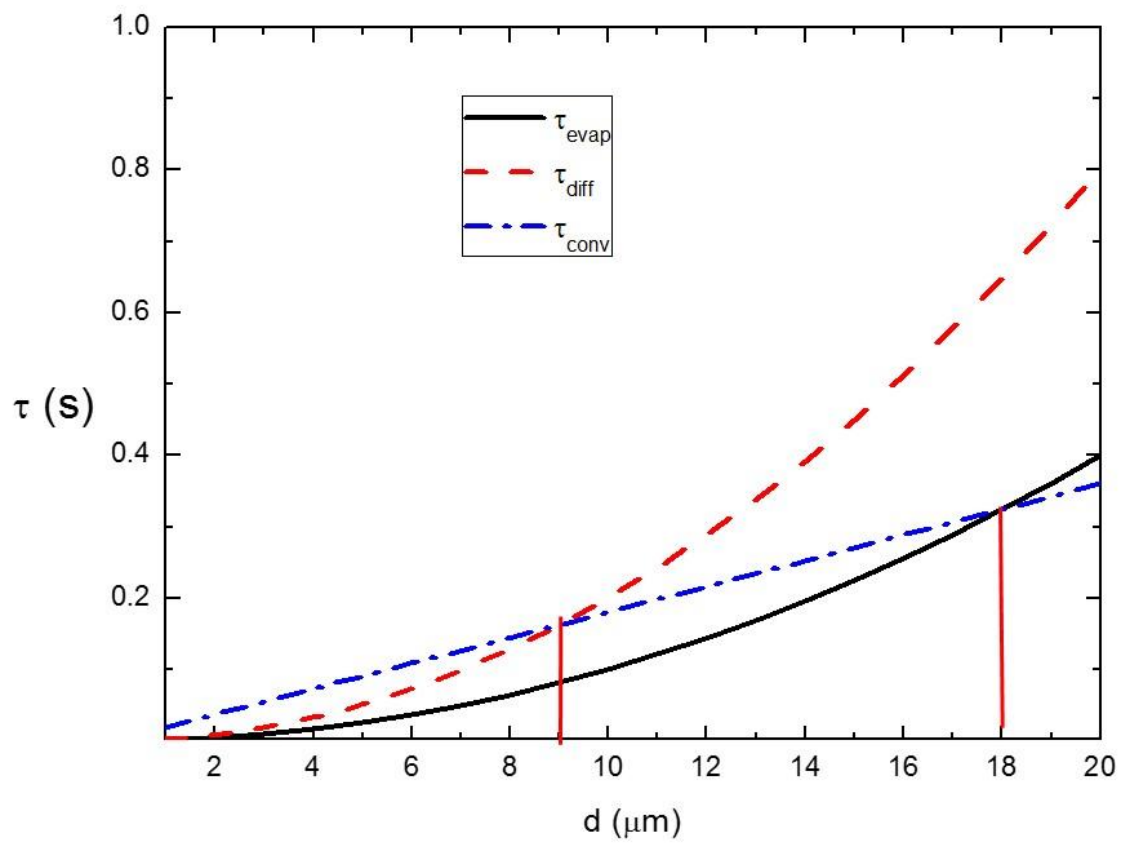

Figure 4 - Time scales of various transport processes as functions of droplet size; $d_{\mathrm{p}}=$ $100 \mathrm{~nm}\left(D_{\mathrm{pl}}=10^{-11} \mathrm{~m}^{2} / \mathrm{s}\right), U_{\infty}=5.5 \mathrm{~cm} / \mathrm{s}$, and $K=10^{-9} \mathrm{~m}^{2} / \mathrm{s}$

It can be said from the above discussion that depending on the droplet size, internal circulation might play (i) an important role in particle redistribution during evaporation (for $d_{\mathrm{o}}>9 \mu \mathrm{m}$ ) or (ii) no role (for $d_{\mathrm{o}}>9 \mu \mathrm{m}$ ) in the morphology of the final particle aggregate. However, such discussions are based on the gradient diffusion occurring only in one direction, which in the absence of internal circulation would cause particle to diffuse radially inward. Caution must thus be exercised in that, as the streamline pattern of Fig. 3 suggests, the particle concentration gradient is not monotonic along the droplet radius; this is particularly true for $\tau_{\text {diff }}>\tau_{\text {conv }}$ (i.e., $d_{\mathrm{o}}>$ $18 \mu \mathrm{m})$, where the streamline pattern resembles the iso-concentration contours.

For the conditions given above, combining Eqns. (5), (7), and (8) leads to

$$
\frac{\tau_{\text {evap }}}{\tau_{\text {diff }}}=\frac{50 D_{\mathrm{pl}}}{K}
$$


and

$$
\frac{\tau_{\text {conv }}}{\tau_{\text {diff }}}=\frac{\mathrm{d}_{\mathrm{o}} / V_{0}}{V_{0}}=\frac{1000 d_{\mathrm{o}} / U_{\infty}}{0.02 d_{\mathrm{o}}^{2} / D_{\mathrm{pl}}}=\frac{5 \times 10^{4} D_{\mathrm{pl}}}{U_{\infty} d_{\mathrm{o}}}
$$

In [1], where no internal circulation was considered, the $P e$ is defined as

$$
P e \equiv \frac{K}{8 D_{\mathrm{pl}}}
$$

and Eqn. (16) can then be rewritten as

$$
\frac{\tau_{\text {evap }}}{\tau_{\text {diff }}}=\frac{6.25}{P e}
$$

Equation (19) shows that the ratio of evaporation and diffusion time is independent of the droplet size, as it can be expected since it is also related to the non-dimensional $P e$ on the right-hand side of Eqn. (19) and since both the evaporation and diffusion time scales are proportional to $d^{2}$. The morphologies of the dried particles in the evaporation process are determined by the Peclet number. For $P e \gg 6.25$,

$$
\tau_{\text {evap }} \ll \tau_{\text {diff }}
$$

Therefore, for $P e \gg 6.25$, the evaporation occurs faster than particle can redistribute within the droplet, forming shells early and eventually hollow aggregates when the liquid is completely vaporized.

As shown by Eqns. (13) - (15), $P e_{\text {eff }}$ is determined by the relatively magnitudes of $\tau_{\text {diff }}$ and $\tau_{\text {conv }}$. Equation (17) can be used to determine the droplet diameter, denoted as $d_{1}$, for $\tau_{\text {conv }}=$ $\tau_{\text {diff: }}$

$$
d_{1}=\frac{5 \times 10^{4} D_{\mathrm{pl}}}{U_{\infty}}
$$


For $d>d_{1}, \tau_{\text {diff }}>\tau_{\text {conv }}$ particle migration within the droplet is dominated by internal circulation; for $d>d_{1}$, internal circulation is the dominant mechanism. Throughout the evaporation process, the general approach to dealing with particle redistribution is to include both internal circulation, arising from non-zero $U_{\infty}$, and $D_{\mathrm{pl}}$. For the $R e=5$ case here discussed, if the particle is silica with a $30 \mathrm{~nm}$ diameter, then $d_{1} \approx 14 \mu \mathrm{m}$. Many commercially available atomizers produce droplet diameters exceeding $14 \mu \mathrm{m}$ and droplets studied in laboratory setting are often of the size of $100 \mu \mathrm{m}-1 \mathrm{~mm}$ range. It might be concluded that droplets containing small particles even in relative weak convective environment (e.g., $\left.U_{\infty}=5.5 \mathrm{~cm} / \mathrm{s}\right)$ the internal circulation within a droplet cannot be ignored. With this in mind, the following section describes the effort to develop a model to account how coupled effects of particle mobility and internal circulation determine particle distribution and shell formation.

\subsection{Theoretical model with internal convection}

In developing a multicomponent droplet evaporation model that accounts for the internal vortex circulation, a simplified model was proposed [10] based on the assumption that there exists a symmetrical Hill's spherical vortex inside the droplet. Eslamian and Ashgriz [11] adopted the analysis for the evaporation of droplet containing insoluble solutions. The simulation results provide good insight in predicting the morphology of the dried particle aggregates. The present study extends these analyses to liquid suspension to study the effect of the internal circulation on the evaporation of droplets containing particles. Results of particle distribution as a function of time, time to shell formation, and shell diameter are sought.

For the evaporation of droplets containing particles at a given temperature and pressure, assume that the particles are initially evenly distributed within the droplet and that evaporation is quasi-steady. Using the streamline as the coordinate, mass concentration is only a function of the stream function. Therefore, the liquid phase mass concentration with strong internal circulation is governed by the following one-dimensional equation, which in non-dimensional form is written as $[10,11]$ : 


$$
\frac{\partial Y_{1}}{\partial \tau}=\frac{\phi}{L e} \frac{\partial^{2} Y_{1}}{\partial \phi^{2}}+\left(\frac{1}{L e}+C \phi\right) \frac{\partial Y_{1}}{\partial \phi}
$$

where $Y_{1}$ is the liquid mass fraction, $d \tau=\left(\alpha_{1} / r_{\mathrm{s}}^{2}\right) d t$, with $\alpha_{1}$ being the diffusivity of the liquid, $L e\left(\equiv \alpha_{\mathrm{l}} / D_{\mathrm{pl}}\right.$, with $D_{\mathrm{pl}}$ being the Eistein-Stokes diffusivity) is the liquid phase Lewis number, and the dimensionless stream function $\phi$ is expressed based on Hill's vortex approximation given by Eqn. (1). Le is also the ratio of the particle diffusion to liquid diffusion time scales. In another word, a small/large value of $L e$ implies large/small particle mobility relative to the liquid molecular mobility.

For the one-dimensional analysis, $\phi$ can be expressed to be, by incorporating Eqn. (1),

$$
\phi \equiv 1-4 \psi_{\mathrm{i}}(1+\sigma) /\left(U_{\infty} \cdot r_{\mathrm{s}}^{2}\right)=1-4 s^{2}\left(1-s^{2}\right) \sin ^{2} \theta
$$

where $s=r / r_{\mathrm{s}}$. Therefore, $\phi=1$ for $s=0$ (center of droplet), $s=1$ (droplet surface), or $\theta=0$ or $\pi$. Equation (24) also indicates that the vortex center (i.e., zero velocity and zero $\phi$ ) is located at $\theta=\pi / 2$ and $s=1 / \sqrt{2}$, with the later coordinate remaining the same as that shown in Fig. 2. Because of the elimination of $\sigma$-dependence of $\phi$ in Eqn. (24), one expect a symmetric circulation vortex, where $Y_{1}=Y_{1}(\tau, \phi)$. The result of $\phi$ is shown in Fig. 5. 


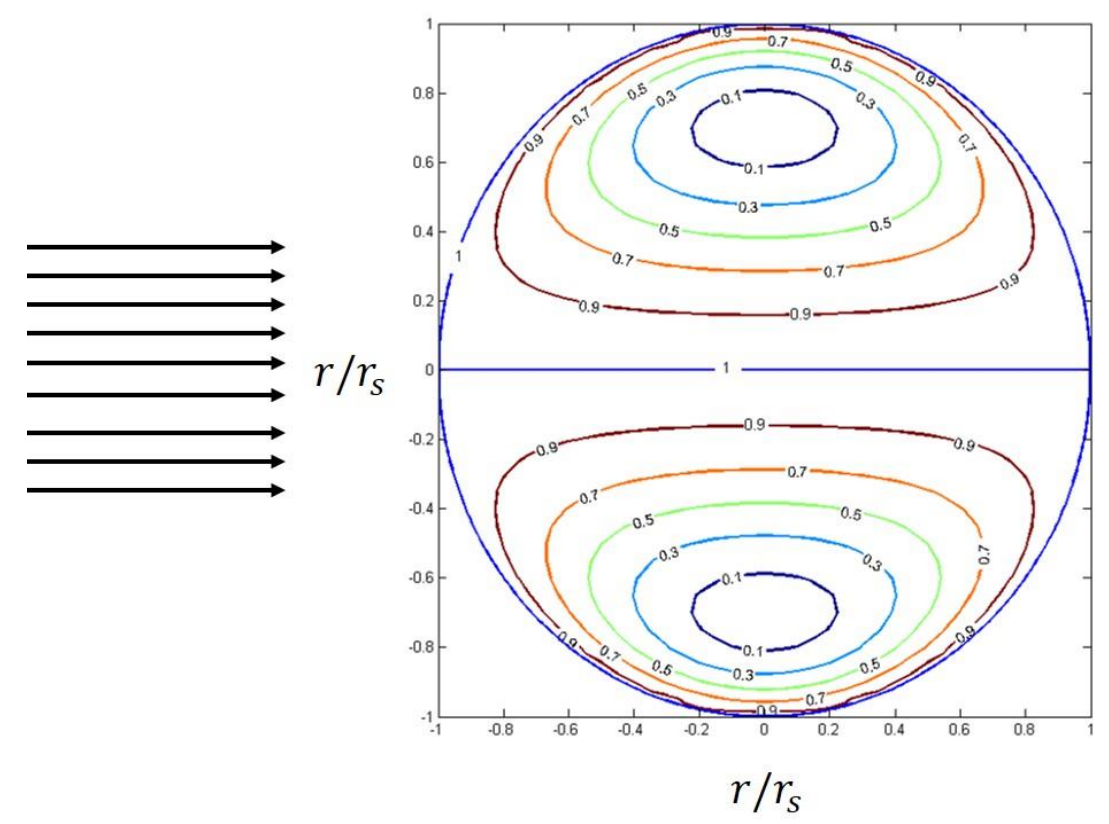

Figure 5 - Stream-function $(\phi)$ contour within the droplet with internal circulation on a meridian plane of the spherical droplet

In Eqn. (23), $C$ relates to the droplet evaporation rate and is given by $[10,11]$

$$
C=2\left(\frac{1}{s}\right)^{\frac{3}{2}} \frac{d}{d \tau}(s)^{\frac{3}{2}}
$$

The initial and boundary conditions for Eqn. (23) are:

$$
\begin{aligned}
& \tau=0: Y_{1}=Y_{10} \\
& \phi=0: \frac{\partial Y_{1}}{\partial \phi}=0 \\
& \phi=1: \frac{\partial Y_{1}}{\partial \phi}=f_{l}
\end{aligned}
$$

where $Y_{\mathrm{lo}}$ is the initial liquid phase mass fraction and $f_{\mathrm{l}}$ is given by 


$$
f_{\mathrm{l}}=\frac{L e C_{\mathrm{pl}}\left(Y_{\mathrm{ls}}-1\right)}{16 \pi r_{\mathrm{s}} \lambda_{\mathrm{l}}} \dot{m}
$$

where $C_{\mathrm{pl}}$ and $\lambda_{\mathrm{l}}$ are, respectively, the liquid phase heat capacity and thermal conductivity, and $Y_{\mathrm{ls}}$ is the liquid mass fraction at the droplet surface.

Similar to the discussion provided by Aggarwal et al. [21], for $\sigma \ll 1$ the velocity boundary layer near the droplet surface is very small compared to $r_{\mathrm{s}}$; that is, the internal circulation in the droplet core region is essentially inviscid. Under this condition, the analysis of the liquid phase would result in axi-symmetric streamline and stream-function pattern, slightly different from that depicted in Fig. 2, which is asymmetric with respect to a meridian plane.

By incorporating the evaporation rate constant from Eqn. (6), Eqn. (25) can be re-written as

$$
C=\frac{3 K}{2 \alpha_{1}}
$$

$C$ is thus the ratio of evaporation rate to the liquid phase thermal diffusivity. It is related to Peclet number defined by Eqn. (18) [paper \#1] in the following manner:

$$
C=\frac{3 K}{2 \alpha_{1}}=\frac{3}{2} \cdot \frac{K}{D_{\mathrm{pl}}} \cdot \frac{D_{\mathrm{pl}}}{\alpha_{1}}=\frac{3}{2} \cdot \frac{P e}{L e}
$$

Therefore $C$ represents the combined effects of evaporation rate and relative importance of liquid and particle diffusivities. Equation (23) is numerically solved by the time and space discretization method to obtain the particle concentration distribution at any given time. The computation is stopped when the particle volume fraction $Y_{\mathrm{pvs}}$ at the droplet surface reaches the maximum value $\left(Y_{\mathrm{pvs}}=0.6\right)$ and forms a shell [paper \#1].

It is important to note the difference between the current derivation and solution of Eqn. (23). First, the current definition of $P e$, by Eqn. (18), refers to particle diffusivity $\left(D_{\mathrm{pl}}\right)$, while in [10, $11]$ it is only for the liquid phase where the Peclet number is the effective value $\left(P e_{\text {eff }}\right)$ resulting 
from strong circulation that leads to Eqn. (23), with $P e_{\text {eff }}$ being smaller than $P e$ without the effect of circulation. Under the strong circulation assumption, the particle diffusion rate in the crossstream direction is small compared to its stream-wise convection rate. Secondly, the current equation to solve, Eqn. (23), differs from those in [Tong/Sirignano] in that in the current study only one species of the multi-component system is capable of vaporizing; once the evaporation rate is known, the particle concentration can be calculated. Thirdly, to directly illustrate the effect of the evaporation rate on the first-stage evaporation with internal circulation, the value of $K$ is assumed, as shown in Table 1. In [11], the evaporation rate can be derived from the temperature fields in both the gas and liquid phases, which in turn were obtained in solving a separate, but similar, equation to Eqn. (23) where Lewis number is not a factor. Further, the Lewis number for common liquids is on the order of a few tens $(\approx 30$ in [22]), while Lewis number for nano-size particles are on the order of $10^{-5}$ (for the conditions given in Table 1), depending strongly on the particle size.

Table 1 - Initial values for parameters of spherical silica particle in water droplet

\begin{tabular}{|c|c|c|c|c|c|c|c|}
\hline$\overline{d_{\mathrm{o}}(\mathrm{mm})}$ & $K\left(\mathrm{~m}^{2} / \mathrm{s}\right)$ & $Y_{\mathrm{vi}}$ & $\rho_{\mathrm{p}}\left(\mathrm{g} / \mathrm{cm}^{3}\right)$ & $d_{\mathrm{p}}(\mathrm{nm})$ & $\alpha_{1}\left(\mathrm{~m}^{2} / \mathrm{s}\right)$ & $D_{\mathrm{pl}}\left(\mathrm{m}^{2} / \mathrm{s}\right)$ & Le \\
\hline \multirow[t]{2}{*}{1} & $10^{-9}$ & $0.144^{\mathrm{a}}$ & 2.09 & 30 & $1.43 \times 10^{-7}$ & $1.43 \times 10^{-11}$ & 10,000 \\
\hline & & $0.024^{\mathrm{b}}$ & & Various $^{\mathrm{b}}$ & Various ${ }^{b}$ & Various ${ }^{b}$ & up to $10,000^{b}$ \\
\hline
\end{tabular}

${ }^{\mathrm{a}}$ For Section 3.1 calculations

${ }^{\mathrm{b}}$ For Sections 3.2 and 3.3 calculations

Since the solution of Eqn. (23) deals with mass fraction, the following conversion is necessary to account for the particle volume fraction distribution throughout the droplet, $Y_{\mathrm{pv}}$, previously used [1] for determining the time for shell formation, which occurs at $Y_{\mathrm{pvs}}=0.6$, with $Y_{\mathrm{pvs}}$ denoting the particle volume fraction at the droplet surface. It is easily shown that

$$
Y_{\mathrm{pv}}=\frac{\left(1-Y_{1}\right) / \rho_{\mathrm{s}}}{\left(1-Y_{1}\right) / \rho_{\mathrm{s}}+Y_{\mathrm{l}} / \rho_{1}}
$$

or 


$$
Y_{\mathrm{l}}=\frac{\left(1-Y_{\mathrm{pv}}\right) / \rho_{\mathrm{l}}}{\left(1-Y_{\mathrm{pv}}\right) / \rho_{\mathrm{l}}+Y_{\mathrm{pv}} / \rho_{\mathrm{s}}}
$$

where $Y_{\mathrm{pv}}, Y_{\mathrm{p}}, \rho_{\mathrm{l}}$, and $\rho_{\mathrm{s}}$ denote the particle volume and mass fractions and liquid and solid phase densities, respectively. Substituting the result of $Y_{p}$ from Eqns. (23) and (26) into Eqn. (30), one obtains the instantaneous particle volume fraction throughout the droplet, including that at its surface. These results allows the determination of time and droplet size when the shell is formed, signaling the end of the first-stage evaporation/drying.

\section{Results and Discussion}

\subsection{Effect of Internal Recirculation on Particle distribution}

Figure 5 shows the stream function contour within the droplet in the case of strong internal circulation, using the parameters shown in Table 1 for $L e=10,000$ for silica nano-particles (30 $\mathrm{nm}$ diameter) in water droplet having an initial diameter $d_{\mathrm{o}}=1 \mathrm{~mm}$. Therefore $d_{\mathrm{o}}>d_{1}=$ $14 \mu \mathrm{m}$ calculated in Sec. 2.1, which satisfies the strong circulation assumption. These parameter provides a Peclet number $P e \approx 70$, which is similar to that for ethanol droplet [1,3]. Note that the value of $L e$ for many liquids, including water, is on the order of a few tens. The value of $L e$ listed in Table 1 is that based on the liquid $\alpha_{l}$ particle diffusivity, $D_{\mathrm{pl}}$, which is about three orders of magnitude smaller than liquids. Note again that for very thin boundary layer in the liquid phase $(\sigma \ll 1)$ the stream-function pattern is symmetric with respect to the equatorial and meridian planes. Also $\phi=1$ for $s=1$ (at the droplet surface), $s=0$ (center of droplet), or $\theta=0$ and $\pi$ (i.e., along the axis of symmetry). Due to symmetry, $\phi=0$ along a circle with $r=r_{s} / \sqrt{2}$ on a meridian plane passing $\theta=\pi / 2$ and $\theta=3 \pi / 2$ (with angle $\theta$ as defined in Fig. 2).

To illustrate the effect of internal circulation on particle distribution, it is useful to first consider the particle concentration of an evaporating droplet without internal circulation. As shown in [1], the particle number density as a function of radial location and time is 


$$
Y_{\mathrm{pv}}(s)=\mathrm{C}_{1} C_{\mathrm{m}} \frac{\exp \left(P e \cdot s^{2} / 2\right)}{3 \int_{0}^{1} s^{2} \exp \left(P e \cdot s^{2} / 2\right) d s}
$$

where $\mathrm{C}_{1}$ is the conversion factor between volume and number densities described in [1] and $C_{m}$ is the average number density within the droplet that can be calculated as a function of time:

$$
C_{\mathrm{m}}=C_{\mathrm{o}}\left(\frac{d_{\mathrm{o}}}{d}\right)^{3}
$$

with $C_{\mathrm{o}}, d_{\mathrm{o}}$, and $d$ are, respectively, the initial particle number concentration, droplet diameter, and the instantaneous droplet diameter. It can be seen that the particle concentration decays in an exponential manner from the droplet surface $(s=1)$ to center $(s=0)$, where the concentration is minimum, and that for large $\mathrm{Pe}\left(=K / 8 D_{\mathrm{pl}}\right)$ the particle concentration at the surface is substantially higher than elsewhere in the droplet and the shell forms early during the evaporation as $Y_{\mathrm{pvs}}=$ $Y_{\mathrm{pv}}(s=1)=0.6$ is rapidly established [1].

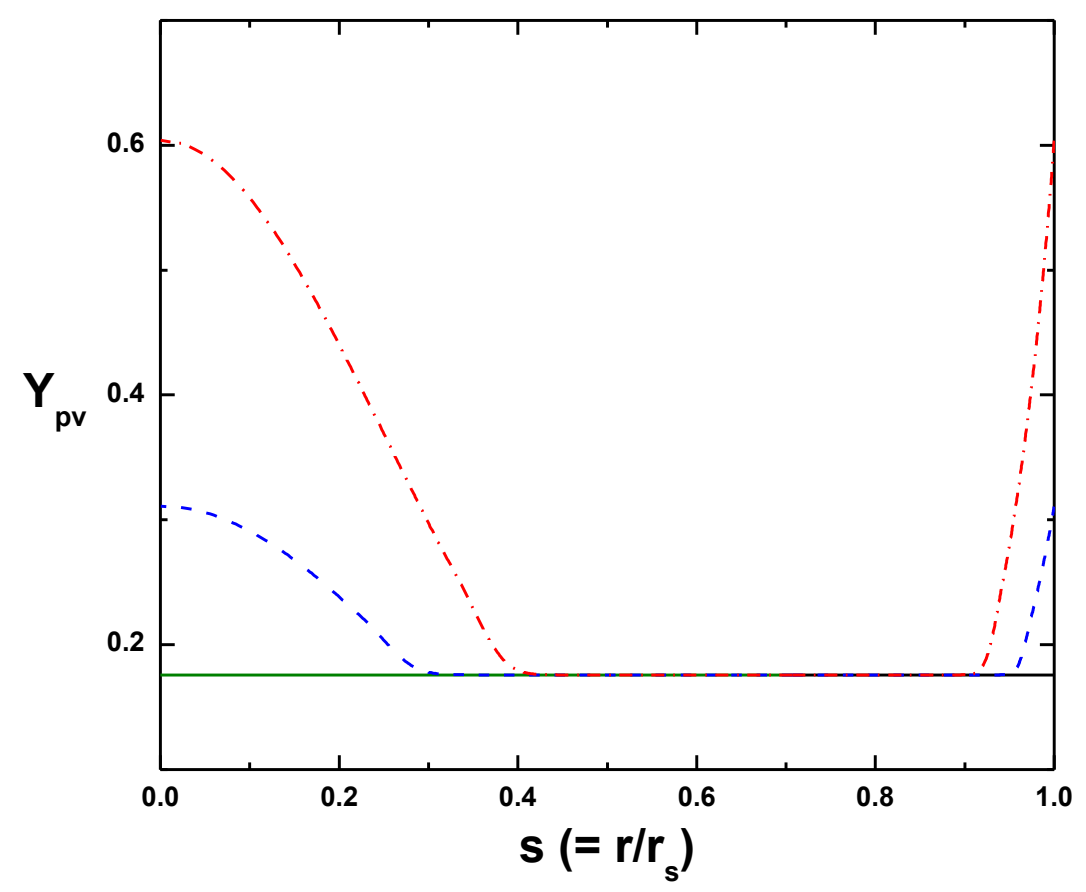

Figure 6 - Variation of the particle volume fraction (using parameters in Table 1) $\left(Y_{\mathrm{pvi}}=\right.$ $0.144)$ 
For a droplet with internal circulation, the particle volume fraction as a function of $t$ and $s$ (= $\left.r / r_{\mathrm{s}}\right)$ is shown in Fig. 6. At $t=0$ the particles are evenly distributed within the droplet and the initial volume fraction $\left(Y_{\mathrm{pvi}}\right)$ is uniform at $Y_{\mathrm{pvi}}=0.144$. For $t=0^{+}$, convection is set in motion and the particle volume fraction becomes non-uniform, with local maximum values of $Y_{\mathrm{pv}}$ occurring at $s=0$ and $s=1$; these two locations correspond to the droplet center and the surface of droplet. Because of vaporization, the value of $Y_{\mathrm{pv}}$ at the droplet surface will increase and because of the strong circulation, such an increased value is spread through out the streamline $\phi=$ 1, which as shown in Fig. 6 are located at $s=0$ and $s=1$. The non-uniformity increases as droplet evaporation proceeds. As can be seen in Fig. 6, at $t=5.7$ seconds, the maximum value is $Y_{\mathrm{v}} \approx 0.32$ and the region for $Y_{\mathrm{pv}}=Y_{\mathrm{pvi}}=0.144$ has shrunk to $s \approx 0.31-0.91$. At $t=$ 11.4 seconds, the maximum value is $Y_{\mathrm{pv}} \approx 0.6$ and the uniform region with $Y_{\mathrm{pv}}=Y_{\mathrm{pvi}}=0.144$ has further shrunk to $s \approx 0.42-0.96$.

The double-peak nature of $Y_{\mathrm{pv}}$ is qualitatively and distinctively different from that depicted by Eqn. (32) for droplets without internal circulation. From Fig. 6 one sees that the two local maxima are nearly equal at each of the two time steps, while $Y_{\mathrm{pv}}$ monotonically increases with $s$ in the absence of internal circulation. This result suggests that particles are being carried around by internal circulation within the droplet without diffusing away from the streamline between $s=0$ and $s=1$, consistent with the strong circulation assumption. One might also see that for $t=$ 11.4 seconds the maximum value of $Y_{\mathrm{pv}}$ at $s=0$ is slightly larger than that at $s=1$. While the maximum value of $Y_{\mathrm{pv}}$ at $s=1$ cannot exceed $Y_{\mathrm{pvs}}=0.6$, as determined by shell formation and the geometry at the droplet surface [1], $Y_{\mathrm{pv}}$ at $s=0$ is not subject such restrictions and is slightly, although negligibly, larger than $Y_{\text {pvs }}$. It is also seen that as the evaporation progresses, the concentration gradient steepens, as expected because there would be no shell formation without such an event.

The case of $t=11.4$ seconds also signifies the end of the first stage of evaporation/drying when the surface volume fraction $Y_{\text {pvs }}$ reach a value of 0.6 and shell forms. The second stage of evaporation commences for $t>11.4$ seconds, when the shell thickens and the liquid within the shell would have to diffuse through capillary action to the shell surface to evaporate. 
The distribution of $Y_{\mathrm{pv}}$ of Fig. 6 also suggests that during the first stage of evaporation, the particle concentration gradients would cause particles to diffuse, although very slowly compared to the convective circulation, toward $s=1 / \sqrt{2}$. Because of the unsteady nature of both the internal circulation the droplet surface regression, the direction of diffusion should be considered in the sense of the bulk flux. Instantaneous particle movement away from the streamline may result in being included at the droplet surface rather than diffusing toward $s=1 / \sqrt{2}$, which is more likely to occur among smaller particles. Without internal circulation, the diffusion of particle is unidirectional toward the droplet center $(s=0)$ [1]. One might expect the morphologies of dried particle aggregates with and without international would be substantially different.

The current model would suggest that the dried particle aggregate would take the shape of a sphere. One might, however, expect that perfect symmetry to be unlikely, as the particle deposition during the second stage might not be uniform because particles should gravitate toward the bottom of each cavity. The dome region of the upper cavity is expected to be the thinnest among all regions, tending to collapse or cave. This might help to explain the formation of dimples and openings on the otherwise spherical particle aggregates, as shown in Fig. 7 (taken from [**]). The mechanism of such dimple formation would involve detail model of contact mechanics [*] and is beyond the scope of this study.
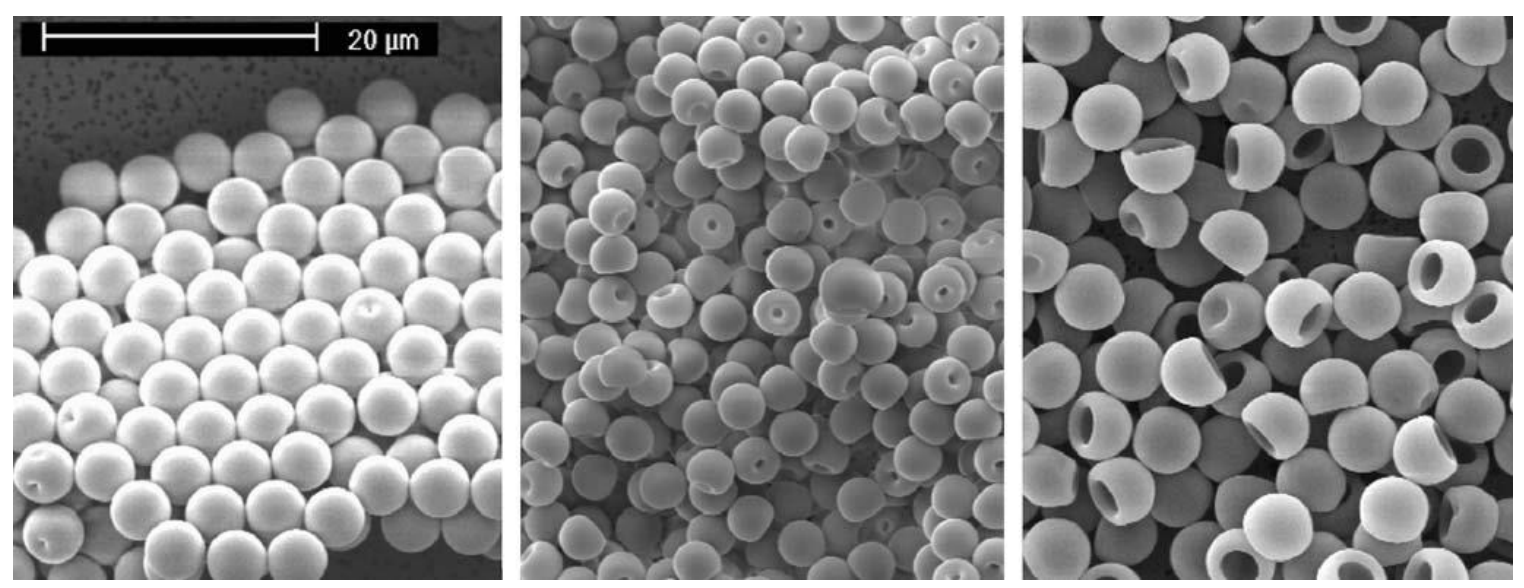

Figure 7 - Morphology of monodisperse glycoprotein particles at Peclet numbers of 2.7, 5.6, and 16.8 (from [23]) 


\subsection{Effect of Particle Mobility on Evaporation}

To reveal the effects of particle mobility on the behavior of first-stage evaporation, $Y_{\mathrm{pvi}}=0.024$, lower than 0.144 from the previous section, has been chosen (see Table 1). This choice prevents the overly early shell formation [1] and allows focusing on effects on particle mobility that can be varied by using particles of various sizes. It can be seen in Fig. 8 for the same conditions for Fig. 6 except the $Y_{\text {pvi }}$ value the time to reach $Y_{\text {pvs }}=0.6$ for shell formation is now $\approx 790$ seconds, longer than 11.4 seconds of Fig. 6. Here mobility is in the form of Lewis number, $L e \equiv \alpha_{1} / D_{\mathrm{pl}}$. For the given pair of water-silica particle, $L e$ is solely determined by the particle radius, $r_{\mathrm{p}}$, as indicated by Eqn. (18), and $L e \sim r_{\mathrm{p}}$; that is, larger $L e$ (or $r_{\mathrm{p}}$ ) is, the less mobile the particle. This section presents the duration of the first-stage evaporation (i.e., time to shell formation, $t^{*}$ ), the shell diameter, $d^{*}$ and the particle distribution $Y_{\mathrm{pvs}}^{*}(=0.6)$ at $t^{*}$--all as functions of Le. Note that the superscript $*$ is used to signify the end of the first-stage evaporation and shell formation.

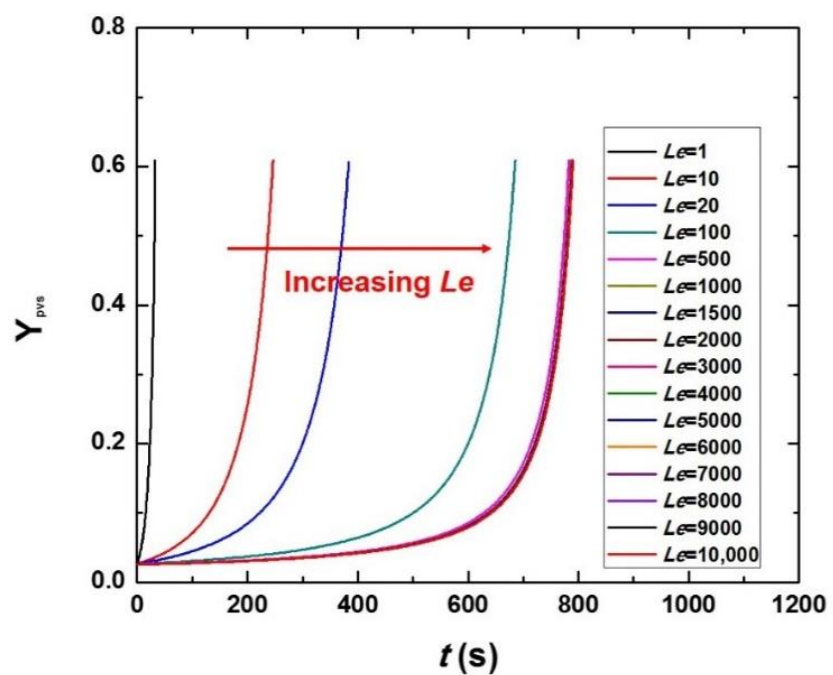

Figure 8 - The surface particles concentration evolution under various Le numbers $\left(Y_{\mathrm{pvi}}=\right.$ 0.024)

Figure 8 shows the evolution of surface particle volume fraction for Lewis numbers ranging from 1 to 10,000 . It is seen that as evaporation proceeds, $Y_{\mathrm{pvs}}$ increases, as expected due to inclusion. For $L e=1$, the inclusion occurs rapidly so that $Y_{\mathrm{pvs}}$ reaches the shell formation value $Y_{\text {pvs }}^{*}$ quickly as shown by the steep $Y_{\text {pvs }}$ curve. For $L e \geq 10$, there exists a slow inclusion period, 
where the value of $Y_{\mathrm{pvs}}$ increases relatively slowly before exhibiting growth in an exponential manner. Upon reaching $Y_{\mathrm{pvs}}^{*}$ the droplet completes its first stage of evaporation (at time denoted by $t^{*}$ ). This "induction" period as a portion of the time to reach $Y_{\mathrm{pvs}}^{*}$ increases with $L e$, suggesting that larger particles tend to escape inclusion at the droplet surface until very closely approaching $t=t^{*}$. A possible explanation lies with the value of $L e$ and $D_{\mathrm{pl}}$. As particles are being convected by the internal circulation, smaller ones (larger $D_{\mathrm{pl}}$ and smaller $L e$ ) diffuse in the cross-streamline direction with larger mobility to reach to, and to be included at, the droplet surface. On the other hand the larger ones (smaller $D_{\mathrm{pl}}$ and larger $L e$ ) remain closely following the streamline and for a longer time and avoid inclusion. However, there exists a limiting value of $L e \geq 500$, as shown in Fig. 8, above which $Y_{\text {pvs }}$ is only controlled by the internal circulation and becomes independent of Le. Under the conditions shown in Table $1, L e \geq 500$ translates to $d_{p} \geq 1.5 \mathrm{~nm}$. The particle associated with $L e=20$ has a size $d_{p}=0.06 \mathrm{~nm}$, which is already smaller than water molecules and would diffuse like liquid, rapidly reaching $Y_{\text {pvs }}^{*}$ and resulting in smaller $t^{*}$, as shown in Fig. 8. It is noted that although with larger $t^{*}$, the fashion in which larger particles are included is no less dramatic than smaller ones in that after a long "induction" time they are included in a similar rapid rate as the latter.

There appears to be a limiting value of $L e$ also applies to $t^{*}$. The result of $t^{*}$ as a function of Le is shown in Fig. 9. The results of both Fig. 8 and 9 suggest an asymptotic value of $t^{*} \approx$ 780 seconds for $L e \geq 500$ under the conditions investigated in this study.

One further distinctive aspect of $t^{*}$ with internal circulation from that in the absence of circulation is worth of noting. Without internal circulation, larger particles (having smaller $D_{\mathrm{pl}}$ and larger $P e[1])$ are less mobile and become included relative early during the evaporation process, resulting in smaller $t^{*}$ [1]. With strong internal circulation, transport of larger particles is dominated by convection and, while they might diffuse away from the streamline, they do so at slower rate compared to smaller particles. They thus also diffuse slower to reach the droplet surface. Consequently, larger particles (with larger values of $L e$ ) tend escape inclusion for a longer time (larger $t^{*}$ ). Such a qualitatively opposite effects of particle size on $t^{*}$, with and without internal circulation, is almost striking! 


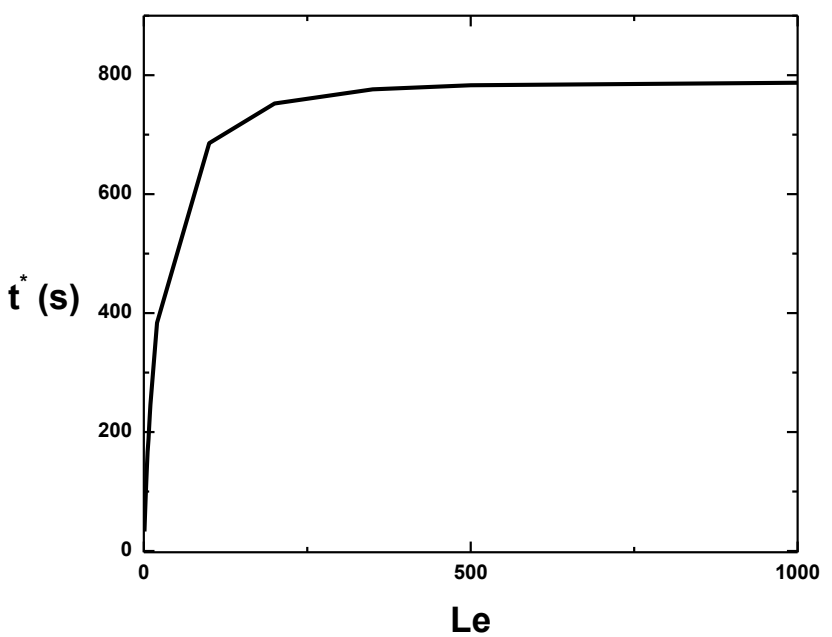

Figure 9 - The duration time of first evaporation stage as a function of $L e\left(Y_{\mathrm{pvi}}=0.024\right)$

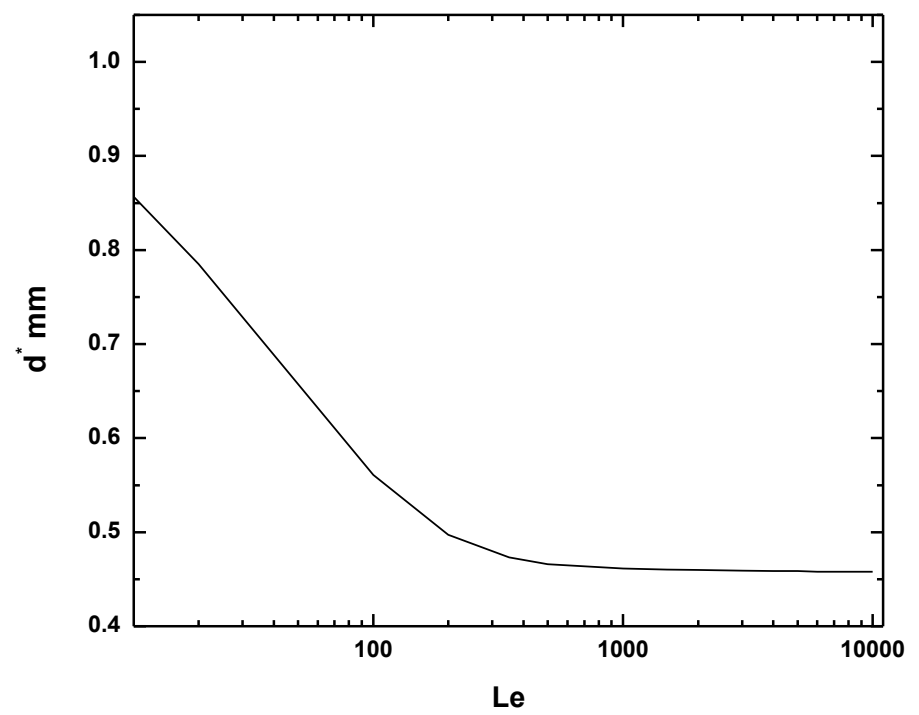

Figure 10 - Droplet size at the end of the first evaporation stage under various $L e$ numbers At d* still > $d_{1}-$ internal circulation still important!! $\left(Y_{\mathrm{pvi}}=\mathbf{0 . 0 2 4}\right)$

Without internal circulation, the shell diameter $d^{*}\left(d\right.$ at $\left.t^{*}\right)$ decreases with increasing $t^{*}$, as shown in [1] that small values of $t^{*}$ means earlier attainment of $Y_{\mathrm{pvs}}^{*}$ at larger droplet diameter. This qualitative trend is also observed for the case with internal circulation, with the present results shown in Fig. 10, where larger values of $L e$ correspond to larger $t^{*}$, as already known from Fig. 9. Nevertheless caution has to be paid to the opposite underlying effects of particle size on $t^{*}$, as 
already revealed. It is further noted that an asymptotic of $d^{*} \approx 0.5 \mathrm{~mm}$ for $L e \geq 500$ mirrors the similar trend of $t^{*}$ shown in Fig. 9.

The fact that $d^{*} \approx 0.5 \mathrm{~mm}>d_{1} \approx 14 \mu \mathrm{m}$ would imply that at the end of the first stage, internal circulation should continue to play an important role. This inference is unlikely to be physically possible. Because of the shell formation, the fluid inside of it would be shielded from the shear stress exerted by the external flow, rendering Eqn. (10) invalid. Such impossibility does not invalidate the results obtained prior to the shell formation, which is the goal of the present study.

\subsection{Effect of Particle Mobility on Particle Distribution}

At the end of first-stage drying, the liquid needs to reach the outer surface of the shell through capillary action to be vaporized. It is desirable to examine the particle distribution at the end of the first stage, i.e., $Y_{\mathrm{pv}}^{*}$ (i.e., $Y_{\mathrm{pv}}$ at $t=t^{*}$ ), as it might suggest the likely morphology of the final particle aggregate. Just like $t^{*}$ and $d^{*}, Y_{\mathrm{pv}}^{*}$ is a function of $L e$, as described in the following by the current model prediction.

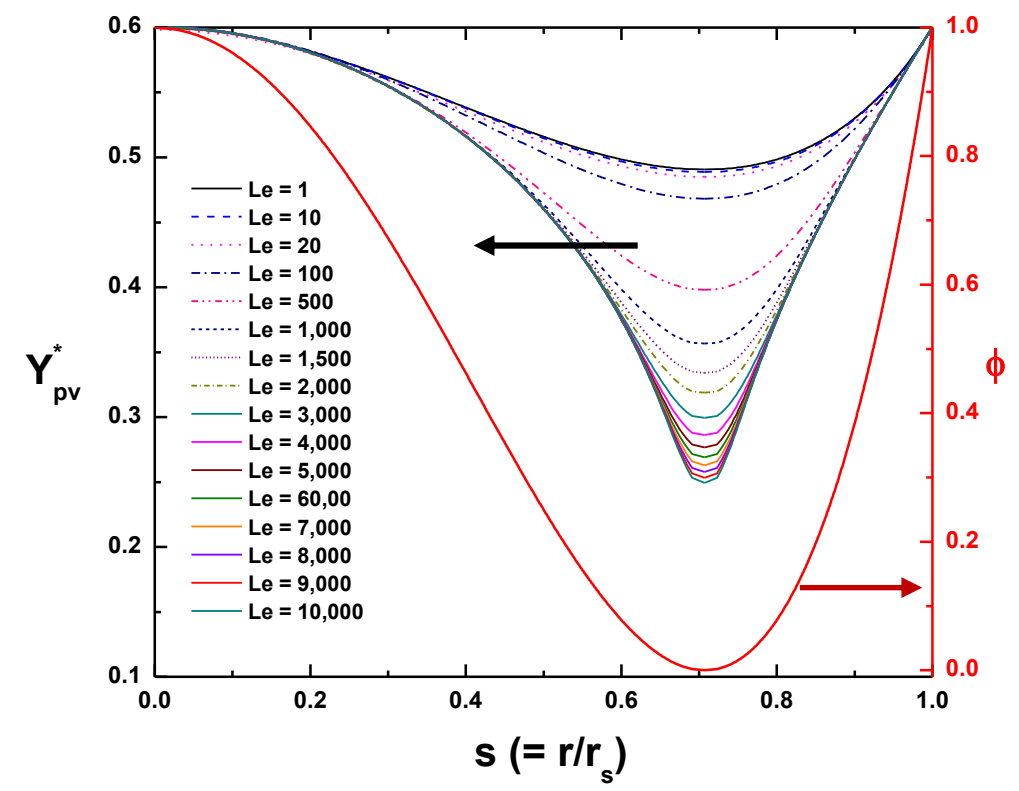

Figure 11 - The particle concentration profile at the end of the first evaporation stage, $Y_{\mathrm{pv}}^{*}(S)$, under various Le numbers $\left(Y_{\mathrm{pvi}}=0.024\right)$ 
The results of $Y_{\mathrm{pv}}^{*}(s)$ for $L e=1-10,000$ are shown in Fig. 11, which was obtained for $\theta=$ $90^{\circ}$. Also plotted in Fig. 11 is $\phi(s)$, to reveal the effect of internal circulation in addition to that of $L e$. Several observations can be made: First, for all values of $L e$ investigated, $Y_{\mathrm{pv}}^{*}$ has a minimum located at $s=1 / \sqrt{2}$, the center of the circulation vortex, where $\phi=0$. This result follows from the earlier discussion of results of Fig. 6, that the high concentration and large value of $Y_{\mathrm{pv}}$ are found near the droplet surface (i.e., $\phi=1$ ) and spreads due to strong circulation, resulting in local maxima of $Y_{\mathrm{pv}}$ at $\phi=1(s=1$ and $s=0)$ and minimum at $\phi=0(s=1 / \sqrt{2})$. Such a pattern of persists throughout and to the end of the first stage. This result is in contrast to the case without internal circulation, where minimum $Y_{\mathrm{pv}}^{*}$ is located at the center of the droplet, as described by Eqn. (32).

Second, the magnitude of $Y_{\mathrm{pv}}^{*}(s)$ over the entire range of $s=0-1$ decreases with increasing $L e$ (due to decreasing particle mobility associated with large size). In particular, the minimum $Y_{\mathrm{pv}}^{*}$ (at $s=1 / \sqrt{2}$ ) is the smallest for the largest $L e$ investigated. This is attributed to the ability of large mobility/small size to smooth out concentration gradients, leading to larger magnitudes of $Y_{\mathrm{pv}}^{*}$ for smaller $L e$. In other words, smaller particles (with larger $D_{\mathrm{pl}}$ and larger $L e$ ) are more capable of diffusing perpendicular to the circulating flow, thus being more able to diffuse, due to the concentration gradient, to the center region of the vortex with particles. The value of $Y_{\mathrm{pv}}^{*}$ throughout the droplet (now covered by a shell) is larger than the initial $Y_{\mathrm{pvi}}=0.024$, suggesting that, particles of all sizes investigated were able to diffuse over the period of $t=0-t^{*}$.

Third, the particle aggregate would likely take the shape of a sphere with two cavities, each corresponding to $Y_{\mathrm{pv}}^{*}$ (at $s=1 / \sqrt{2}$ ) located symmetrically with respect the axis of symmetry of Fig. 5. These cavities should become more pronounced with increasing Le. For comparison. note that without internal recirculation, both $Y_{\mathrm{pv}}^{*}$ and $Y_{\mathrm{pv}}$ monotonically decrease from droplet surface to center, as indicated again by Eqn. (32) and one single cavity at center should result within the final particle aggregate. 
It is of interest to map $Y_{\mathrm{pv}}^{*}$ vs. $\phi$ shown in Fig. 11, with the result presented in Fig. 12. It is seen that for small values of $L e\left(\leq 100\right.$, i.e., $d_{\mathrm{p}} \leq 0.3 \mathrm{~nm}$ ), $Y_{\mathrm{pv}}^{*}$ decreases linearly with $\phi$, from 0.6 at the droplet surface to approximately 0.5 at the center of the vortex. That is, $\partial Y_{\mathrm{pv}}^{*} / \partial r$ is constant in both the regions from the vortex center toward the droplet center and droplet surface. Considering the vortex center being at $s=1 / \sqrt{2}$, the constant value of $\partial Y_{\mathrm{pv}}^{*} / \partial r$ outward from the vortex center is a factor of $(\sqrt{2}+1)$ of that in the inward region. For $L e>100\left(d_{\mathrm{p}}>0.3 \mathrm{~nm}\right)$, $\partial Y_{\mathrm{pv}}^{*} / \partial r$ is not constant in either region; however for a given $L e$, the $(\sqrt{2}+1)$ factor remains true. These results imply different morphologies of the dried aggregates of particle having different sizes. As an example, for droplets containing larger particles the final aggregate would have less compact regions around $s=1 / \sqrt{2}$ than elsewhere. For similar reasons, larger values of $L e$ also leads to less compact regions around $s=1 / \sqrt{2}$ compared to smaller $L e$, although their shells form with the same $Y_{\mathrm{pvs}}^{*}=0.6$, as shown in Fig. 12.

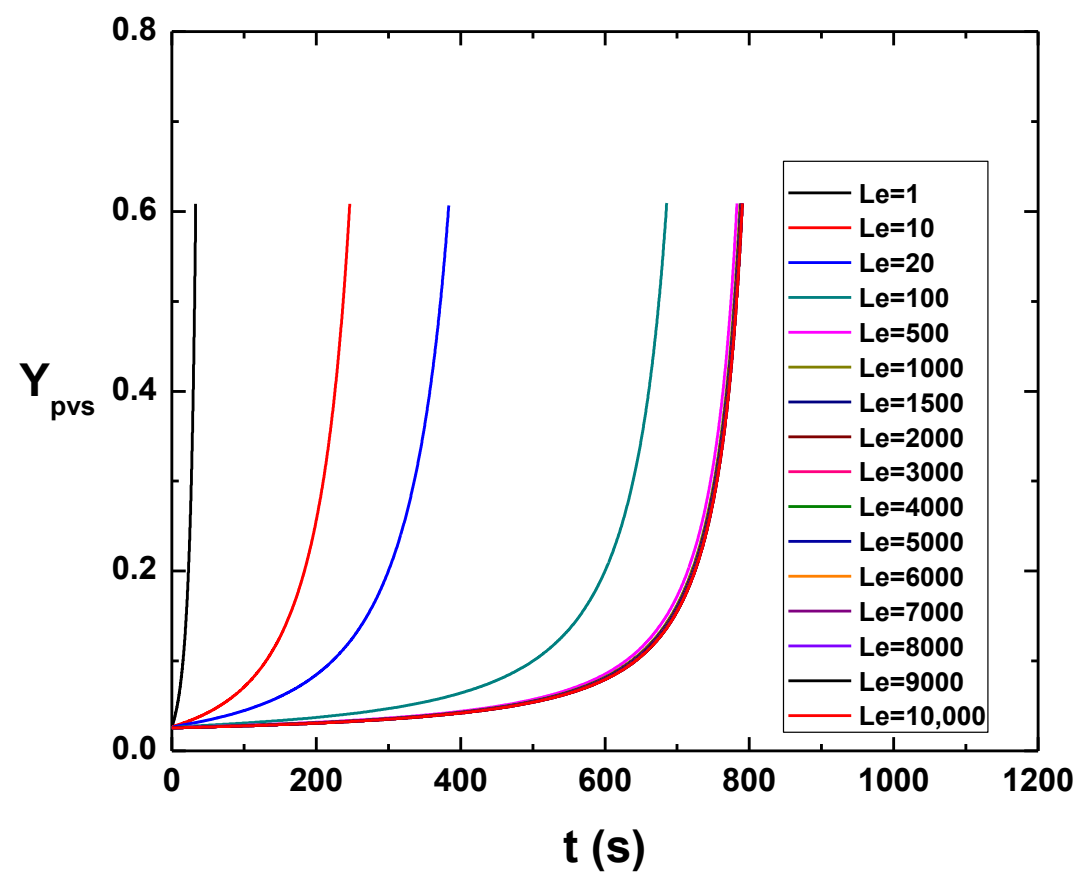

Figure 12 - The particle concentration profile as a function of $\phi$ at the end of the first evaporation stage under various Le numbers $\left(Y_{\text {pvi }}=0.024\right)$ 


\subsection{Effect of Initial Particle Concentration $\left(Y_{\mathrm{nvi}}\right)$}

In Sections 3.2 and 3.3, the value of $Y_{\text {pvi }}$ was reduced for the purpose of prolonging the first-stage evaporation and $t^{*}$, where the intended observations were made. In this section the detailed effect of $Y_{\mathrm{pvi}}$ is presented. Of particular interest are the results of $t^{*}$ and $d^{*}$, shown respectively in Figs. 13 and 14.

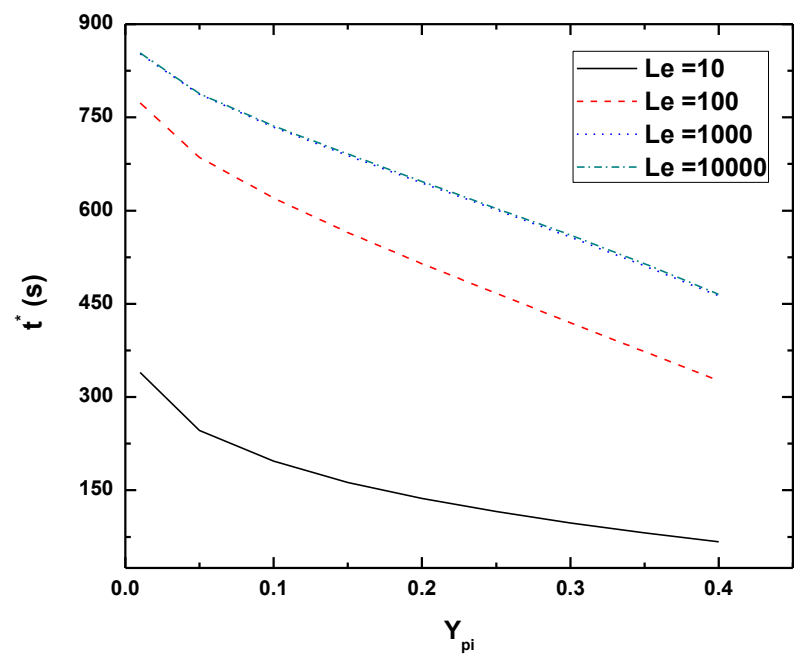

Figure 13 - The duration time of the first evaporation stage under various initial particles mass fraction

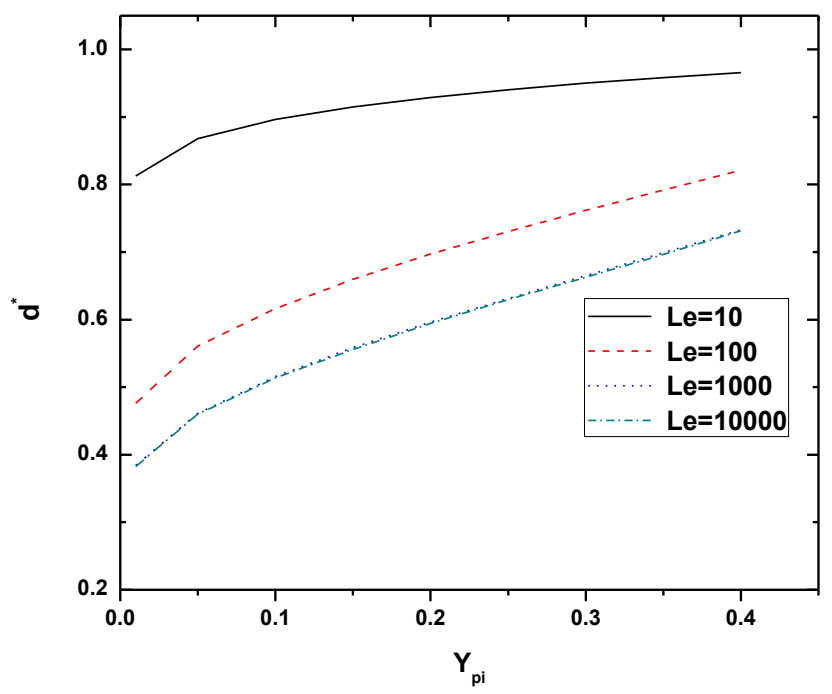

Figure 14 - The droplet size at the end of the first stage under various initial particle mass fraction $\left(Y_{\mathrm{pvi}}=\mathbf{0 . 0 2 4}\right)$ 
Figure 13 shows the effect of $Y_{\text {pvi }}$, in conjunction with $L e$, on $t^{*}$. It can be seen that for a given $L e, t^{*}$ decreases with increasing $Y_{\mathrm{pvi}}$, similar to droplets without internal circulation [1] in that larger $Y_{\text {pvi }}$ naturally leads to larger particle inclusion rate on the droplet surface increases. For a given $Y_{\text {pvi }}, t^{*}$ increases with $L e$ but become insensitive to sufficiently larger $L e$. To explain this relative sensitivity to $L e$, recall that particles with small $L e$ (larger $D_{\mathrm{pl}}$ ) are also capable of diffusional movement normal to the circulation flow. They thus will offer themselves to be included at the droplet surface at faster rates than particles with larger $L e$. This difference result in larger $t^{*}$ for smaller $L e$. With sufficiently larger $L e$, the circulation flow becomes more dominant over the diffusional movement, causing the insensitivity of $t^{*}$ to $L e$. The decrease in $t^{*}$ with increasing $D_{\mathrm{pl}}$ is opposite to that observed for droplets without internal circulation, as noted earlier for results of Fig. 9.

Following similar reasoning, $d^{*}$ increases with increasing $Y_{\mathrm{pvi}}$ and with decreasing $L e$. These results are shown in Fig. 14, where the insensitivity of $d^{*}$ to $L e$ for sufficiently large Le (i.e., $\geq$ 1,000 in Fig. 14) is also seen. Combining results of Figs. 13 and 14 again indicates that $d^{*}$ increases with decreasing $t^{*}$, whether the latter is caused by increasing $Y_{\mathrm{pvi}}$ or $D_{\mathrm{pl}}$. Also recall again for comparison that for droplet without internal circulation (i) increasing $Y_{\text {pvi }}$ leads to an decrease in $t^{*}$ and $d^{*}$, and (ii) increasing $D_{\mathrm{pl}}$ (decreasing Peclet number) leads to an increase in $t^{*}$ and a decrease in $d^{*}$.

\subsection{Effect of Evaporation Rate Constant $(K)$ and Peclet Number (Pe)}

Equation (23) takes into account of the effect of evaporation rate constant $K$, or the Peclet number $\left(P e=K / D_{\mathrm{pl}}\right)$, through coefficient $C=3 K / 2 \alpha_{1}=3 P e / 2 L e$, where $K$ is the evaporation rate constant. For a given $L e, C$ is solely dependent on $P e$ and Eqn. (23) indicates that $L e$ and $C$ can be independent varied. $L e=1,000$ is chosen so that $t^{*}$ and $d^{*}$ are not $L e$-dependent and the effect of $K$ (and thus of $P e$ ) can be clearly demonstrated. The range of $P e$ chosen (shown in Table 2) corresponds to previously investigated values of $P e$ without internal circulation, so that results of the two distinctly cases might be made. In short Table 1 contains a fixed $K$ value with $L e$ varied, while Table 2, a fixed $L e$ value with $K$ varied. The initial droplet diameter is chosen to be $d_{\mathrm{o}}=100$ 
$\mu \mathrm{m}$ as an appropriate example, so tha $d_{\mathrm{o}}>d_{1}$ and internal circulation is the dominant mechanism for particle migration, as calculated for water droplets containing $30 \mathrm{~nm}$ silica particles by Eqn. (21).

Table 2 - Parameters for investigating effect of $K$ and $P e$

\begin{tabular}{|c|c|c|c|c|c|}
\hline$d_{\mathrm{o}}(\mathrm{mm})$ & $Y_{\mathrm{vi}}$ & $L e$ & $K\left(\mathrm{~m}^{2} / \mathrm{s}\right)$ & $C$ & $P e$ \\
\hline 0.1 & 0.024 & 1,000 & $\begin{array}{l}10^{-9} \\
5 \times 10^{-9} \\
10^{-8} \\
5 \times 10^{-8} \\
10^{-7}\end{array}$ & $\begin{array}{l}0.01 \\
0.05 \\
0.1 \\
0.5 \\
1\end{array}$ & $\begin{array}{l}6.7 \\
33.3 \\
66.7 \\
333.3 \\
666.7\end{array}$ \\
\hline
\end{tabular}

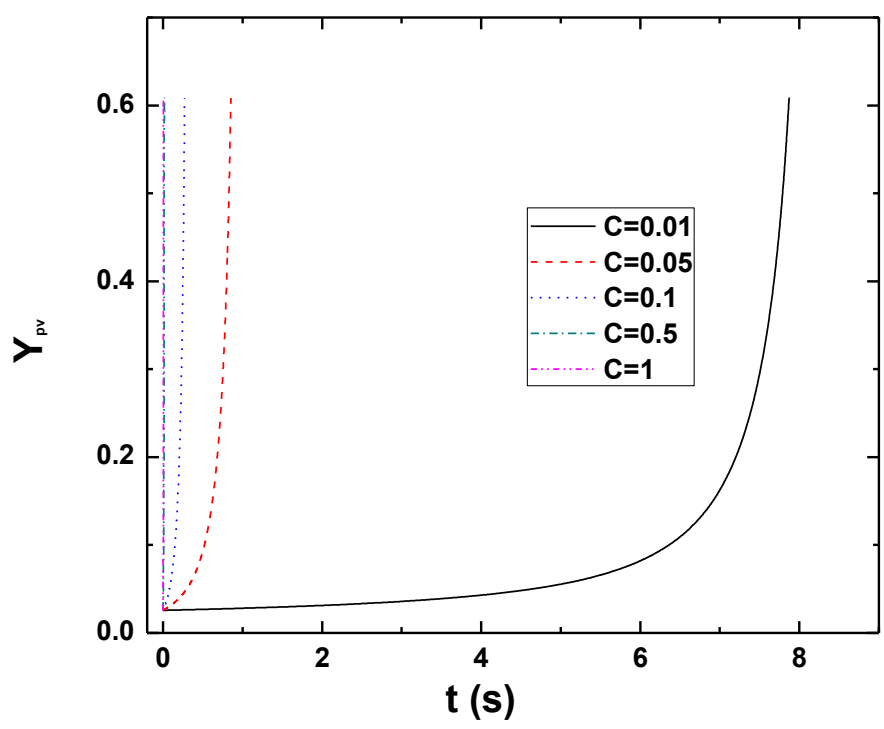

Figure 15 - The surface particles concentration history under various values of $C\left(Y_{\mathrm{pvi}}=\right.$ 0. 024; $L e=1,000$ )

Figure 15 shows the time evolution of $Y_{\text {pvs }}$ for the values of $C$ of Table 2. It is seen that $Y_{\text {pvs }}$ increases with time more sharply for larger $C$ numbers, as the associated higher droplet regression rates (i.e., larger $K$ ) causes larger inclusion rate of particles at the droplet surface to reach $Y_{\mathrm{pvs}}^{*}$, the end of the first stage. However for $C \geq 0.5$ ( $P e \geq 333.3$ ), the time to reach $Y_{\text {pvs }}^{*}$ according to results of Fig. 15, does not appear to change further. Further calculations for $t^{*}$ with finer 
increments of $C$ are presented in Fig. 16, where $t^{*}$ reaches an asymptotic value for $C \geq 0.2(P e \geq$ 133.4).

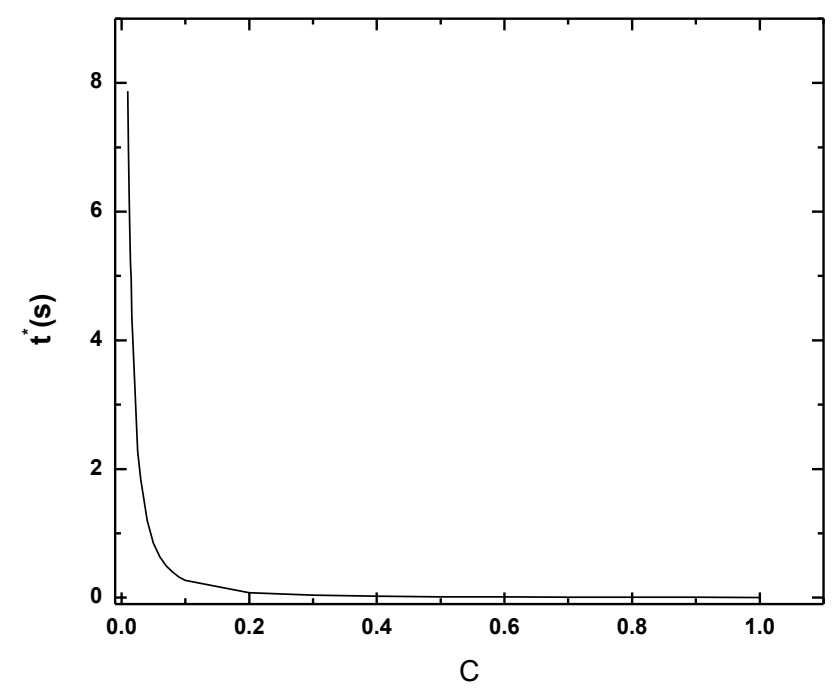

Figure 16 - The duration time of the first evaporation stage as a function of $C\left(\boldsymbol{Y}_{\mathbf{p v i}}=\right.$ 0. 024; $L e=1,000$ )

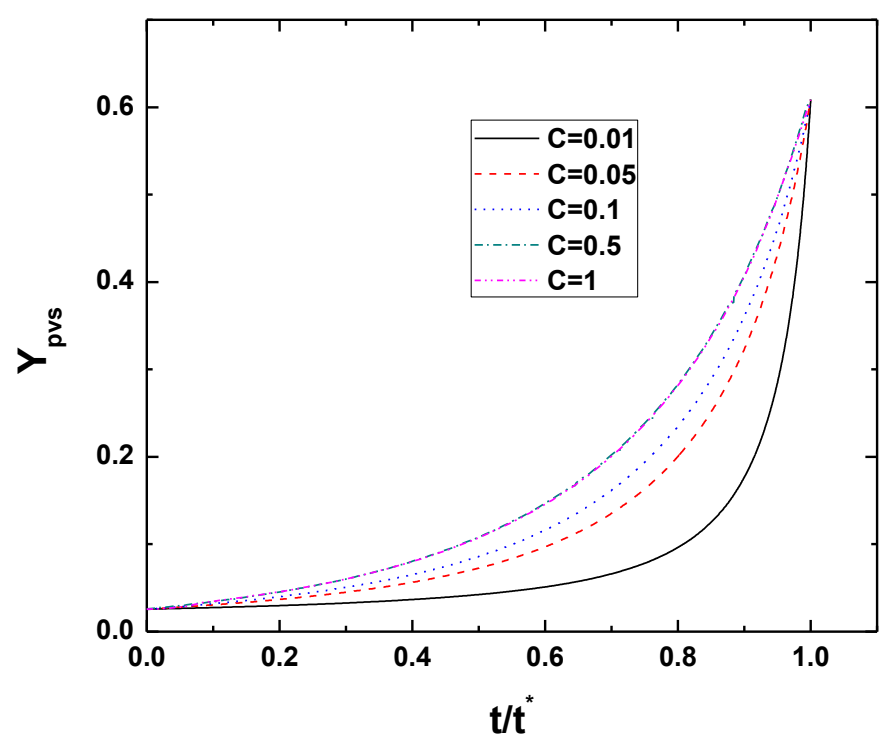

Figure 17 - The evolution of $Y_{\mathrm{pvs}}$ under various values of $C\left(Y_{\mathrm{pvi}}=0.024 ; L e=1,000\right)$

Evaporation with large values of $P e$ is associated with processes such burning of fuel droplets, where for heavy fuel oil (HFO) the morphology of final ash particles determine how easily airborne they are. One might also expect the value of $L e$ of HFO to be large, so that a combination 
of large values of $P e$ and $L e$ leads to small $t^{*}$, large $d^{*}$, and less dense inside of the aggregate, that is, easily air-borne ash particles (or large aerodynamic diameters).

The time history of $Y_{\mathrm{pvs}}$ is plotted against the dimensionless time, $t / t^{*}$, in Fig. 17. It is seen that for a given $t / t^{*}, Y_{\mathrm{pvs}}$ is higher for larger $C$ values (i.e., higher evaporation rates and larger $P e)$. For smaller values of $C$, the inclusion rate is smaller and particles are allowed more time to redistribute besides being circulated (see Fig. 16) and $Y_{\mathrm{pvs}}^{*}$ is reached more rapidly (steeper slope of $Y_{\mathrm{pvs}}$ in Fig. 17) toward the end of the first stage. This result is qualitatively the same as the case without internal circulation [paper \#1], in that with more time for redistribution the value of $Y_{\mathrm{pvs}}^{*}$ is reached in a more dramatic manner.

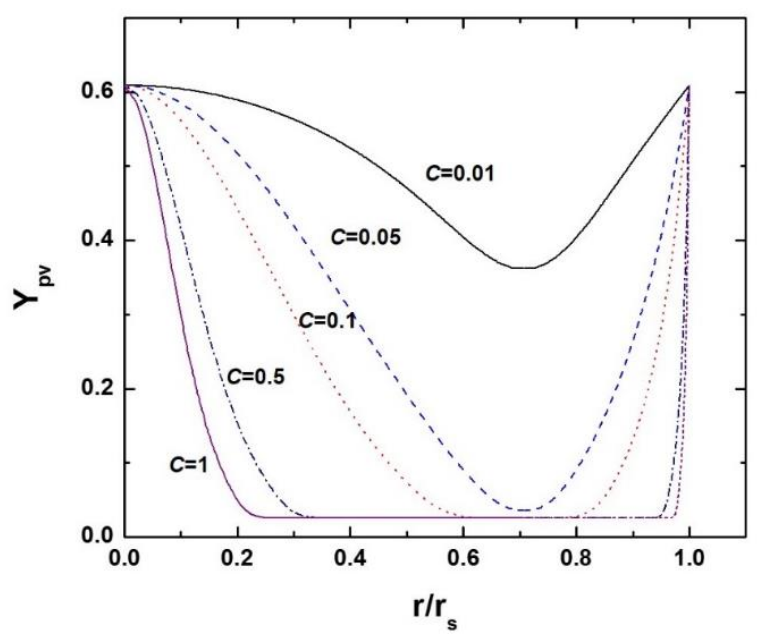

Figure $18-Y_{\mathrm{pvs}}$ at the end of the first evaporation stage under various values of $C$ ( $Y_{\mathrm{pvi}}=$ $0.024 ; L e=1,000$ )

The particle concentration distribution at $t^{*}$, i.e., $Y_{\mathrm{pv}}^{*}$, is shown in Fig. 18. Similar to the results of Fig. 17, larger values of $C$ allow particles less time to redistribute, which in turn results in the region around the vortex center relatively devoid of particles. It can be seen in Fig. 18 that for $C \geq 0.1$, there exists a region near the vortex center where remains $Y_{\mathrm{vi}}(=0.024)$ at $t^{*}$ and the larger $C$ is, the larger the extent this region is. For $C=1$, this region occupies nearly $80 \%$ of the radial extent (for $r / r_{\mathrm{s}}$ approximately equal to $0.2-1$ ) of the droplet. Since larger values of $C$ are associate with rapid evaporation (large $K$ and Pe), leading to smaller $t^{*}$ and thus allowing less time for particles to redistribute. Therefore, the lager $C$ is, the hollower and the larger the cavity is. 


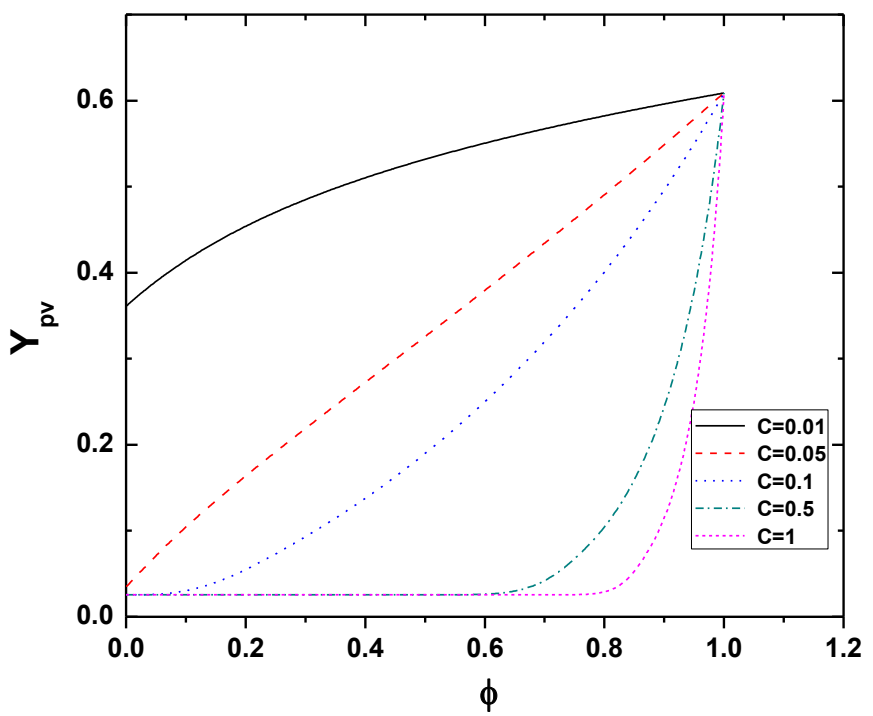

Figure 19 The particle concentration profile as a function of phi at the end of the first evaporation stage under various $\boldsymbol{C}$

In contrast to Fig. 12 (where $C$ is fixed for demonstrating effects of Le), Fig. 19 shows the particle concentration in the stream function field with $L e=1,000$ to study the effect of $C$. For smaller $C$ (and $K$ ), the characteristic time of the internal flow is smaller than that of the droplet shrinking. The particle distribution is driven by the internal flow. As $C$ increases, the particles distribution profile is changed due to the decrease of characteristic time of droplet shrinking and increase in time for particle redistribution. Consider the two extreme cases of Fig. 19 for comparison - for $C=1$ particles are not allowed sufficient time for redistribution resulting in a wide range of where $Y_{\mathrm{pv}}$ remains the initial value of $Y_{\mathrm{pvi}}=0.024$, while for $C=1, Y_{\mathrm{pv}}$ reaches a value approximately 0.36 at $\phi=0$ (i.e., $s=1 / \sqrt{2}$ ).

\subsection{Some comparisons with multi-component droplets with soluble contents}

For evaporation of multi-component droplets, more studies have been conducted for soluble contents (for more recent work in this area see, for example, $[7,24,25]$ ) than for nanoparticles. Some of these works deal with solidification (or solid shell formation) of one component when the concentration of it at the droplet surface exceed the saturation level. Although the shell formation kinetics is more complex for soluble content than for nanoparticles, some similarities deserve 
notice. First, the shell formation in both cases are due to the attainment of saturation concentration, at the droplet surface, of one of the component. Secondly, when evaporation rate is increased, either due to a parametric increase in $P e$ ([1] and the present study) or simply due to a more heated or lower-humidity environment [25], the time to form shell $\left(t^{*}\right)$ in both case decreases, which, in turns, leads to increased shell diameter $\left(t^{*}\right)$. Third, increasing the initial concentration $\left(Y_{\mathrm{pvi}}\right)$ leads to smaller $t^{*}$ and larger $d^{*}$.

Many of these studies using soluble contents were conducted using acoustically levitated, where internal circulation occurs mostly likely with one single vortex due to droplet vibration and rotation [17], rather than the two symmetric vortices described by Hill [12]. However, it is striking to note the similar results of the first stage evaporation/drying for both cases with soluble and insoluble solids. Because of the single vortex within acoustically levitated droplets, the morphology can be expected to differ from that result from Hill's vortex that forms due to external convective flows.

\section{Conclusion}

Based on the assumptions of strong internal circulation and symmetric Hill' vortex for the circulation, the one-dimensional governing equation, Eqn. (23), for particle distribution during and up to the shell formation possess the effects of convection, particle mobility (Lewis number, $L e$ ), and relative importance of evaporation rate and particle diffusivity (Peclet number, $P e$, or the parameter $C$ in Eqn. (23)). Solution and discussion of it lead to the following results.

(a) Due to the strong internal circulation, particle distribution is dominated by convection and possesses twin peaks (at the droplet surface and center), with the minimum particle concentration located at the non-dimensional location of $s=1 / \sqrt{2}$ from the center. Such a characteristic persists throughout the first stage of drying, on which this study focuses. This is opposite to droplets without internal circulation, where the concentration distribution is and remains monotonically decreasing toward the droplet center. As a consequence, the final dried particle aggregate are likely to have cavities with internal 
circulation located at $s=1 / \sqrt{2}$, while in the absence of circulation the cavity is at droplet center $(s=0)$.

(b) With large particle mobility (and small Lewis number), internal circulation leads to a delay of particle shell formation (at time $t^{*}$ ), while in the absence of circulation, the opposite occurs. However because the shell diameter $\left(d^{*}\right)$ decreases with increasing $t^{*}$, the trend of $d^{*}$ vs. $t^{*}$ appears to be qualitatively the same, although the reasons for the resultant $t^{*}$ are distinctly different in cases with and without internal circulation.

(c) It is found that both $t^{*}$ and $d^{*}$ reach their respective asymptotical values for sufficiently large value of $L e$ arising from low particle mobility (approximately 500 in this study), suggesting that further decreasing particle mobility has no effect on $t^{*}$ and $d^{*}$. A similar result for droplet without internal circulation was reported [paper\#1]; in that case Pe is the relevant parameter and the asymptotic result was reached for $P e \leq 3$, suggesting further increasing particle mobility has no further effect on $t^{*}$ and $d^{*}$.

(d) For a given circulation strength, increasing evaporation rate $(K)$ or $P e$ reduces $t^{*}$, a similar finding as for droplet without internal circulation.

Acknowledgment - Part of this work was conducted while RHC was on IPA assignment at the U.S. National Science Foundation (NSF) as a program director in the Division of Chemical, Bioengineering, Environmental, and Transport Systems, from 2012 to 2015). The support by NSF was through Individual Research Days. WD acknowledges partial support from NSF (CMMI1335295 and CMMI-1454406). Both WD and RHC were previously with Department of Mechanical and Aerospace Engineering, University of Central Florida. The authors appreciate Dr. Weiwei Yang's help with plotting many of the figures presented in this paper. 


\section{Reference}

[1] Y. We. W. Deng, and R.-H. Chen, "Effects of insoluble nano-particles on nanofluid droplet evaporation, International Journal of Heat and Mass Transfer, Vol. 97, 725-734 (2016).

[2] G. Derkachov, K. Kolwas, D. Jakubczyk, M. Zientara, and M. Kolwas, "Drying of a Microdroplet of Water Suspension of Nanoparticles: from Surface Aggregates to Microcrystal," Journal of Physical Chemistry C, vol. 112, no. 43, pp. 16919-16923 (2008).

[3] Y. Gan and L. Qiao, "Evaporation characteristics of fuel droplets with the addition of nanoparticles under natural and forced convections," International Journal of Heat and Mass Transfer, vol. 54, no. 23-24, pp. 4913-4922 (2011).

[4] R.-H. Chen, T. X. Phuoc, and D. Martello, "Effects of nanoparticles on nanofluid droplet evaporation," International Journal of Heat and Mass Transfer, vol. 53, no. 19-20, pp. 3677-3682 (2010).

[5] W.J. Gerken, A.V. Thomas, N. Koratkar, and M.A. Oehlschlaeger, "Nanofluid pendant droplet evaporation: experiments and modeling," International Journal of Heat and Mass Transfer, vol. 74, 263268 (2014).

[6] F.R. Newbold and N.R. Amundson, "A model for evaporation of a multi-component droplet," AIChE Journal, vol. 19, 22-30 (1973)

[7] H. Grosshan, M. Griesing, M. Monckedieck, T. Hellwig, B. Walther, S.R. Gopireddy, R. Sedelmayer, W. Pauer, H.-L. Moritz, N.A. Urbanetz, E. Gutheil, "Numerical and experimental study of the drying of nicomponent droplets under various drying condition," International Journal of Heat and Mass Transfer, vol. 96, 97-109 (2016).

[8] C.K. Law, "Recent advances in droplet vaporization and combustion," Prog. Energy. Combust. Sci., vol. 8, pp. 171-201 (1982).

[9] W.A. Sirignano, "Theory of multicomponent fuel droplet vaporization," Archives of Thermodynamics and Combustion, 9(2): 231-47 (1978).

[10] A.Y. Tong and W.A. Sirignana, "Multicomponent droplet vaporization in a high temperature gas," Combustion and Flame. 66(3): 221-35 (1986).

[11] M. Eslamian and N. Ashfriz, "Modeling of particle formation by spray pyrolysis using droplet internal circulation," International Communications in Heat and Mass Transfer, vol. 33, 863-871 (2006).

[12] M.J.M. Hill, "On a spherical vortex," Proceedings of the Royal Society of London, vol. 55(331-335): 219-224 (1894).

[13] J.S.C.R. Hadamard, "Mouvement permanent lent d'une sphere liquid et visqueuse dans un liquid visqueux," CR Acad. Sci., 152(25): 1735-1738 (1911).

[14] F.H. Garner and R.B. Keey, "Mass transfer from single solid spheres - I: Transfer at low Reynolds numbers,"Chemical Engineering Science. 9(2): 119-29 (1958).

[15] F.H. Garner and J.J. Lane, "Mass transfer to drops of liquid suspended in a gas stream. Trans. Inst. Chem. Eng., 37:167-172 (1959).

[16] S.C. Wong and A.C. Lin, "Internal temperature distributions of droplets vaporizing in hightemperature convective flows," Journal of Fluid Mechanics, vol. 237, 671-687 (1992).

[17] Z.L. Yan, W.J. Xie, and B. Wei, "Vortex flow in acoustically levitated drops," Physics Letters A, vol. 375, 3306-3309 (2011).

[18] S. Prakash and W.A. Sirignano, "Theory of convective droplet vaporization with unsteady heat transfer in the circulating liquid phase," International Journal of Heat and Mass Transfer, vol. 23(3): 253-268 (1980). 
[19] M. Renksizbulut and R.J. Haywood, "Transient droplet evaporation with variable properties and internal circulation at intermediated Reynolds numbers," International Journal of Multiphase Flow, vol. 14(2): 189-202 (1988).

[20] B.P. LeClair, A.E. Hamielec, H.R. Pruppacher, and W.D. Hall, "A theoretical and experimental study of the internal circulation in water drops falling at terminal velocity in air," Journal of Atmospheric Sciences, vol. 29(4). 728-740 (1972).

[21] S.K. Aggarwal, W.A. Sirignano and A.Y. Tong, "A comparison of vaporization models in spray calculations," AIAA Journal, 22(10): 1448-1457 (1984).

[22] A. Makino and C.K. Law, "On the controlling parameter in the gasification behavior of multicomponent droplets," Combustion and Flame, 73(3): 331-336 (1988).

[23] Vehring R. 2008. Pharmaceutical Particle Engineering via Spray Drying. Pharmaceutical Research. 25(5): 999-1022 (2008).

[24] G. Brenn, L.J. Deviprasath, F. Durst, and C. Fink, "Evaporation of acoustically levitated multi-component liquid droplets," International Journal of Heat and Mass Transfer, vol. 50, 50735086 (2007).

[25] S.R. Gopireddy and E. Gutheil, "Numerical simulation of evaporation and drying of a bicomponent droplet," International Journal of Heat and Mass Transfer, vol. 66, 404-411 (2013). 